\title{
When Entanglement Meets Classical Communications: Quantum Teleportation for the Quantum Internet
}

\author{
(Invited Paper) \\ Angela Sara Cacciapuoti, Senior Member, IEEE, Marcello Caleffi, Senior Member, IEEE, Rodney Van Meter, \\ Lajos Hanzo, Fellow, IEEE
}

\begin{abstract}
Quantum Teleportation is the key communication functionality of the Quantum Internet, allowing the "transmission" of qubits without the physical transfer of the particle storing the qubit. Quantum teleportation is facilitated by the action of quantum entanglement, a somewhat counter-intuitive physical phenomenon with no direct counterpart in the classical word. As a consequence, the very concept of the classical communication system model has to be redesigned to account for the peculiarities of quantum teleportation. This re-design is a crucial prerequisite for constructing any effective quantum communication protocol. The aim of this manuscript is to shed light on this key concept, with the objective of allowing the reader: i) to appreciate the fundamental differences between the transmission of classical information versus the teleportation of quantum information; ii) to understand the communications functionalities underlying quantum teleportation, and to grasp the challenges in the design and practical employment of these functionalities; iii) to acknowledge that quantum information is subject to the deleterious effects of a noise process termed as quantum decoherence. This imperfection has no direct counterpart in the classical world; iv) to recognize how to contribute to the design and employment of the Quantum Internet.
\end{abstract}

Index Terms-Quantum Communications, Quantum Internet, Quantum Noise, Quantum Teleportation, Entanglement.

\section{INTRODUCTION}

The interconnection of quantum devices via the Quantum Internet - i.e. through a network enabling quantum communications among remote quantum nodes - represents a disruptive

A.S. Cacciapuoti and M. Caleffi are with FLY: Future Communications Laboratory, Department of Electrical Engineering and Information Technology (DIETI), University of Naples Federico II, Naples, 80125 Italy (E-mail: angelasara.cacciapuoti@unina.it marcello.caleffi@unina.it). The authors are also with the Laboratorio Nazionale di Comunicazioni Multimediali, National Inter-University Consortium for Telecommunications (CNIT), Naples, 80126 Italy. Web: www.quantuminternet.it

Rodney Van Meter is with the Faculty of Environment and Information Studies, Keio University, Fujisawa, Kanagawa 252-0882, Japan (e-mail: rdv@sfc.wide.ad.jp).

Lajos Hanzo is with with the School of Electronics and Computer Science, University of Southampton, Southampton SO17 1BJ, UK (e-mail: lh@ecs.soton.ac.uk.

The work of A.S. Cacciapuoti and M. Caleffi was supported by the project "Towards the Quantum Internet: A Multidisciplinary Effort", University of Naples Federico II, Italy.

L. Hanzo would like to acknowledge the financial support of the Engineering and Physical Sciences Research Council projects EP/Noo4558/1, EP/PO34284/1, COALESCE, of the Royal Society's Global Challenges Research Fund Grant as well as of the European Research Council's Advanced Fellow Grant QuantCom. technology [1]- [5]. Indeed, the Quantum Internet is capable of supporting functionalities with no direct counterpart in the classical world [6]-[10], such as secure communications [11], blind computing [12] $-[14]$, exponential increase of the quantum computing power [3], [5] and advanced quantum sensing techniques [15]. These functionalities have the potential of fundamentally changing markets and industries - such as commerce, intelligence and military affairs.

At a first sight, the design of the Quantum Internet might sound like a trivial task. After all, the number of devices interconnected by the conventional Internet exceeds 17 billion [16], hence connecting few extra quantum devices might not seem like a 'big deal'. However, the laws of quantum mechanics impose unusual constraints on the design of the Quantum Internet.

Specifically, the Quantum Internet facilitates quantum communications among remote nodes by transmitting quantum bits (qubits) - which differ fundamentally from classical bits - or by creating distributed, entangled quantum states with no classical equivalent. A classical bit encodes one of two mutually exclusive states, being in only one state at any time. In contrast, a qubit can be in a superposition of the two basis states (see Sec. III). Hence, while $n$ classical bits are only ever in one of the $2^{n}$ possible states at any given moment, an $n$ qubit register can be in a superposition of all of the possible states [1], [5].

Unfortunately, quantum mechanics does not allow an unknown qubit to be copied or observed/measured [17], [18]. Hence, although we can map a qubit to a photon that can be directly transmitted to a remote node via a fiber link or free space, if the traveling photon is lost due to attenuation or it is corrupted by noise, the associated quantum information cannot be recovered via a measuring process or by re-transmitting a copy of the original information. As a consequence, the techniques mitigating the imperfections imposed on the qubits cannot be directly borrowed from classical communications. Hence the direct transmission of qubits remains limited to relatively short distances at the time of writing in the context of specific applications that can tolerate low transmission success rate, such as Quantum Key Distribution (QKD) and Quantum Secure Direct Communication (QSDC) networks [11], [19]. 
Thankfully 1 , quantum teleportation, originally proposed in [22], constitutes an astonishing strategy for "transmitting" qubits within a quantum network, without the physical transfer of the particle storing the qubit [1], [3], [5], [23], [24]. Quantum teleportation, experimentally verified over substantial distances between 500-1400 kilometers [25], exploits the weird quantum phenomenon represented by the quantum entanglement [17]. Specifically, to realize quantum teleportation a pair of parallel resources are needed. One of these resources is classical: two bits must be transmitted from the source to the destination. The other resource is quantum: an entangled pair of qubits must be generated and shared between the source and the destination. As a consequence, quantum teleportation requires two parallel communication links, a classical link for transmitting the pair of classical bits and a quantum link for entanglement generation and distribution [17].

With this in mind, it appears plausible that the very concept of the classical communication system model, as originally proposed by Shannon in his pioneering contribution [26], has to be redesigned to account for these peculiarities of quantum mechanics. This re-design is a crucial prerequisite for conceiving quantum communication protocols.

The aim of this treatise is to shed light on this key concept, with the objective of allowing the reader:

i) to appreciate the fundamental differences between the transmission of classical information versus the teleportation of quantum information;

ii) to understand the communications functionalities underlying quantum teleportation and to highlight the challenges of turning the vision of the Quantum Internet into reality.

In Sec. II we commence by introducing the preliminaries of quantum mechanics, required for appreciating the fundamental difference between classical and quantum networks.

Then, in Sec. III. we review the quantum teleportation process, by providing the rudimentary mathematical preliminaries, followed by describing some representative schemes conceived for practical entanglement generation and distribution. These basics are crucial for understanding the communication system model proposed in Sec. IV to account for the peculiarities of noiseless quantum teleportation. Then, in Sec. V], we introduce realistic imperfections into the quantum teleportation process.

Specifically, similarly to classical communications, quantum communications are subject to the imperfections imposed by the deleterious effects of the environment. These imperfections are termed as decoherence - a type of quantum noise with no direct counterpart in the classical world. Understanding decoherence is pivotal for the design of efficient quantum communication techniques and protocols. Hence, in Sec. V we highlight the theoretical framework of quantum noise modeling from a communications engineering perspective to allow the reader:

\footnotetext{
${ }^{1}$ For long-distance quantum communications, quantum teleportation relies on quantum repeaters that counteract photon losses and gate errors in a variety of ways, depending on loss rates, memory lifetimes and available resources [5], [11], [20], [21]. Although the details of various repeater design are beyond the scope of this article, in Sec. VII-B we clarify some aspects related to the concept of quantum repeater and entanglement swapping.
}

iii) to recognize that the quantum-domain noise is multiplicative rather than being additive and it exhibits an asymmetric behavion ${ }^{2}$ with respect to the three Cartesian coordinates ${ }^{3}$ representing a qubit.

Indeed, as it will be detailed in Sec. V] decoherence is not the only source of imperfections in the quantum teleportation process. In fact, quantum teleportation relies on a sequence of operations applied to the quantum states, as it will be detailed in the following sections. The imperfections of these operations aggravate the imperfections affecting the quantum teleportation. However, the imperfections accumulated throughout the quantum operations strongly depend on the particular technology adopted for representing a qubit. Hence, in Sec. VI we will report on the results of an extensive campaign of teleportation experiments carried out by using the IBM Q quantum processor [29], with the aim of gaining experimental insights into the cumulative imperfections affecting the teleported qubit at the destination in order to confirm the modeling of the quantum decoherence detailed in Sec. V

Finally, Sec. VII concludes the paper by summarizing the results obtained and by providing a long-term perspective on the design of the Quantum Internet.

\section{A. Outline and Contributions}

By using terminology and concepts tailored for the communications engineering community, the objective of this treatise is to depart from the classical Shannonian communication system model in order to account for the peculiarities of quantum mechanics. Hence we strike up a dialogue and develop a common terminology between the theoretical quantum communication community and the classical communications engineering community. To this aim, in Sec. II] we collected and summarized the fundamental concepts and phenomena of quantum mechanics, followed by Sec. II distilling the preliminaries detailed in classic books such as [17], [18].

Then, in order to allow the reader to appreciate the fundamental difference between classical transmission and quantum teleportation, in Sec. III we review the quantum teleportation process by providing the mathematical model and the description of some representative schemes conceived for practical entanglement generation and distribution.

Based on Secs. II and III in Sec. IV we propose a communication system model for the quantum teleportation process that accounts for the peculiarities of quantum mechanics. In particular, we highlight the communication functionalities of quantum teleportation so as to help readers in the design of the Quantum Internet.

\footnotetext{
${ }^{2}$ As detailed in Sec. V. decoherence may impose different types of errors on a qubit, such as bit-flip errors, phase-flip errors, as well as simultaneous bitand phase-flip errors, while, in the classical domain, only bit-flips may occur. Given the nature of the quantum-domain imperfections, the probability of bitflips and phase-flips tends to be different, regardless of the specific material representing the qubits, as seen in Table 1 of [27] and Fig. 6 of [28], which the authors succinctly refer to as an 'asymmetric' property. In this paper we adopt this terminology but in a broader sense, as it will be clarified in Sec. V

${ }^{3}$ As introduced in Sec. II-H and further detailed in Sec. V there exists a one-to-one mapping between a qubit and a Cartesian vector $\mathbf{r}=\left[r_{x}, r_{y}, r_{z}\right] \in$ $\mathcal{R}^{3}$, known as Bloch vector.
} 
In Sec. V, we describe the potential imperfections of the communication system model developed for quantum teleportation, with an emphasis on the decoherence process. Although decoherence is a widely-studied phenomenon, this section goes beyond the review of existing sources by critically appraising the existing results specifically tailored for the communication engineering community.

Finally, Sec. VI reports on the new results of an extensive campaign of teleportation experiments carried out by using the IBM Q quantum processor. The aim of this section is not only to gain experimental insights into the cumulative imperfections affecting the teleportation process, but also to experimentally verify the modeling of the quantum decoherence developed in Sec. V

\section{BACKGROUND}

In this section, we introduce some preliminaries on quantum mechanics.

\section{A. The Hilbert Space}

According to one of the quantum mechanics postulates, any isolated or closed quantum physical system is associated with a complex Hilbert space ${ }^{4}$ This complex vector space is known as the state space of the system. The system is completely described by its state vector, which is a unit-vector in the system's state space [17], [18].

The simplest quantum mechanical system is the quantum bit (qubit), whose state space is two-dimensional. To characterize a quantum state in the state space, a basis that is orthonormal to this state space has to be chosen. In the following, we adopt the conventional bra-ket notation ${ }^{5}$ for denoting a qubit [5], [17], [28], [30]. The most commonly used basis is the standard (or computational) basis, which corresponds to the convention:

$$
|0\rangle \equiv\left[\begin{array}{l}
1 \\
0
\end{array}\right], \quad|1\rangle \equiv\left[\begin{array}{l}
0 \\
1
\end{array}\right] .
$$

Given the vector space framework postulated by quantum mechanics, the state $|\psi\rangle$ of a qubit can be expressed as a linear combination of the basis states chosen:

$$
|\psi\rangle=\alpha|0\rangle+\beta|1\rangle,
$$

where $\alpha$ and $\beta \in \mathbb{C}$ are complex numbers, known as the amplitudes of the state $|\psi\rangle$. Equation (2) portrays the state $|\psi\rangle$ in a superposition of the two basis states. The condition of $|\psi\rangle$ being a unit-vector, which can be formulated as $\langle\psi|| \psi\rangle=1$, is therefore equivalent to $|\alpha|^{2}+|\beta|^{2}=1$. This condition is also known as the normalization condition of the state vectors [17], [18].

Intuitively, the states $|0\rangle$ and $|1\rangle$ are analogous to the values 0 and 1 that a bit may assume. However, a qubit differs from a classical bit, since the superpositions of the two basis

\footnotetext{
${ }^{4}$ In the finite-dimensional complex vector spaces encountered in quantum computation and information processing, a Hilbert space is equivalent to a vector space with inner product.

${ }^{5}$ The bra-ket notation (also known as Dirac's notation) is a standard notation describing quantum states. In a nutshell, a ket $|\cdot\rangle$ represents a column vector, whereas a bra $\langle\cdot|=| \cdot\rangle^{\dagger}$ represents the conjugate transpose of the corresponding ket.
}

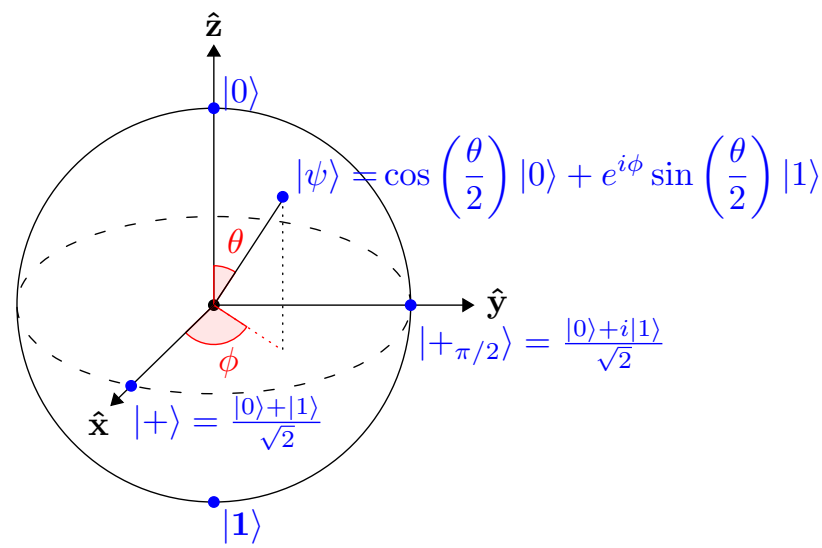

Fig. 1: Bloch sphere: geometrical representation of a qubit in spherical coordinates. A pure state $|\psi\rangle=\alpha|0\rangle+\beta|1\rangle$ is represented by a point on the sphere surface, with $\alpha=\cos \frac{\theta}{2}$ and $\beta=e^{i \phi} \sin \frac{\theta}{2}$.

states in (2) can also exist [17], [18]. Consequently, a classical bit encodes one of two mutually exclusive states, being in only one state at any time. Conversely, a qubit can be in a superposition of the two basis states.

To elaborate a little further, the state of a qubit is often represented geometrically by the Bloch sphere, which is depicted in Fig. 1 and surveyed in more depth in [5], [17], [18], [28]. Specifically, any pure quantum state is represented by a point on the sphere's surface, with $\theta$ and $\phi$ denoting the spherical coordinates. Unfortunately, visualizing the state of more than one qubit is more complicated, since the state space grows exponentially with the number of qubits, as described in Sec. II-D

\section{B. Quantum Measurement}

The amplitudes of the state $|\psi\rangle$ in (2) can be manipulated by quantum gates, as we will see further below, but they also have a bearing on the linkage between the quantum and classical world. In fact, according to one of the postulates of quantum mechanics - namely, the quantum measurement - although a qubit may reside in a superposition of two orthogonal states, when we want to observe or measure its value, it collapses into one of the two orthogonal states [5], [17], [28]. More explicitly, $|\alpha|^{2}$ and $|\beta|^{2}$ uniquely determine the probabilities of obtaining $|0\rangle$ or $|1\rangle$, respectively, by measuring the qubit state on the basis $\{|0\rangle,|1\rangle\}$. Hence the normalization condition may also be further interpreted in the light of $|\alpha|^{2}$ and $|\beta|^{2}$ being probabilities.

After its measurement/observation, the original quantum state collapses to the measured state. Hence, the measurement irreversibly alters the original qubit state. For instance, if the outcome of measuring a superposed qubit is the state zero, the qubit collapses into this specific state and any

\footnotetext{
${ }^{6}$ The measurement of a qubit state may also be carried out in a basis different from that in which the qubit was prepared in [17], [18]. In the above description, for the sake of clarity, we assumed the standard basis also for the measurement.
} 
further measurement will always give the state zero outcome, regardless of the original contribution of the state one to the superposed state [1]. Measurements can be performed in the computational basis described above, corresponding to the $\mathrm{Z}$ axis of the Bloch sphere. It can also be performed in the $|+\rangle$ $/|-\rangle$ basis (X axis), or any axis, such as a diagonal basis (needed to statistically prove the presence of entanglement, below), where the two outcomes represent the opposite points on the Bloch sphere.

The measurement postulate has deep implications on the design of the quantum communication protocols as described below.

\section{Phase}

The angle $\phi$ mentioned above is referred to as the phase of the quantum state [5]. To be more rigorous, the difference between the global phase and relative phase has to be clarified.

Specifically, we say that the state $e^{i \gamma}|\psi\rangle$ is equal to $|\psi\rangle$ up to the global phase factor $e^{i \gamma}$, with $\gamma$ being the global phase. In fact, the statistics of measurement predicted for these two states are the same, being $\left|e^{i \gamma}\right|=1$ [5], [17], [18]. Therefore, from an observational point of view, these two states are identical. Hence, the global phase factors are neglected, since they are irrelevant to the observed properties of the physical system.

However, the relative phase $\phi$ cannot be neglected, and indeed it is critical to quantum computation. Specifically, the relative phase (in the standard basis) of a superposition $|\psi\rangle=\alpha|0\rangle+\beta|1\rangle$ is a measure - in the complex plane of the angle between $\alpha$ and $\beta$, i.e, $\phi: \beta / \alpha=e^{i \phi}|\beta| /|\alpha|$ [18]. A pair of superpositions $|\psi\rangle=\alpha|0\rangle+\beta|1\rangle$ and $\left|\psi^{\prime}\right\rangle=\alpha^{\prime}|0\rangle+\beta^{\prime}|1\rangle$, whose amplitudes have the same magnitudes but differ in the relative phase ${ }^{7}$ represent different states. Furthermore, the role of the relative phase is pivotal in creating the interference patterns exploited for instance in the construction of quantum algorithms. In fact, the state of a quantum system is a wave function that matches Schrödinger's equation. Similar to classical wave mechanics, a pair of waves can interfere, either constructively or destructively, depending on the relative phases of the waves. When the resultant interference is constructive, it enhances the amplitude (hence, probability) of a particular state. By contrast, when it is destructive, it reduces the probability.

\section{Composite Quantum System}

In classical physics, the legitimate states of a system of $n$ objects, whose individual states can be described by a vector in a two-dimensional vector space, can be described by vectors in a vector space of $2 n$ dimensions, i.e. the classical state spaces combine through the direct sum. By contrast, in quantum mechanics, the state space of a composite quantum system made up of $n$ quantum systems, each having states modeled by two-dimensional vectors, is much larger. Indeed, the vector

${ }^{7}$ For instance, $|+\rangle \equiv \frac{|0\rangle+|1\rangle}{\sqrt{2}}$ and $\left|+_{\pi / 2}\right\rangle \equiv \frac{|0\rangle+i|1\rangle}{\sqrt{2}}$ have the same magnitudes, i.e., $|\alpha|=\left|\alpha^{\prime}\right|$ and $|\beta|=\left|\beta^{\prime}\right|$, but they differ by the relative phase of $\pi / 2$. spaces associated with the constituent quantum systems can be combined using their tensor product, which is denoted by $\otimes$, resulting in a vector space of $2^{n}$ dimensions. Hence, if the systems are numbered 0 through $n-1$, and the system having the index $i$ is prepared in the state $\left|\psi_{i}\right\rangle$, then the joint state $|\psi\rangle$ of the resultant composite system is [17], [18]:

$$
|\psi\rangle=\left|\psi_{0}\right\rangle \otimes\left|\psi_{1}\right\rangle \otimes \ldots\left|\psi_{n-1}\right\rangle .
$$

A more compact and readable notation uses $\left|\psi_{0} \psi_{1} \ldots \psi_{n-1}\right\rangle$ to represent $\left|\psi_{0}\right\rangle \otimes\left|\psi_{1}\right\rangle \otimes \ldots\left|\psi_{n-1}\right\rangle$.

By exploiting this notation, if $V$ and $W$ are vector spaces corresponding to a qubit, each having the standard basis of $\{|0\rangle,|1\rangle\}$, then the composite two-qubit system $V \otimes W$ has the basis:

$$
\{|00\rangle,|01\rangle,|10\rangle,|11\rangle\}
$$

Just like a single qubit system, a possible state of a two-qubit system can be in a superposition of the basis states:

$$
|\psi\rangle=\alpha_{0}|00\rangle+\alpha_{1}|01\rangle+\alpha_{2}|10\rangle+\alpha_{3}|11\rangle=\left[\begin{array}{c}
\alpha_{0} \\
\alpha_{1} \\
\alpha_{2} \\
\alpha_{3}
\end{array}\right],
$$

with $\alpha_{0}, \alpha_{1}, \alpha_{2}, \alpha_{3} \in \mathbb{C}:\left|\alpha_{0}\right|^{2}+\left|\alpha_{1}\right|^{2}+\left|\alpha_{2}\right|^{2}+\left|\alpha_{3}\right|^{2}=1$.

Further generalizing this procedure, an $n$-qubit system can be in a superposition of all the $2^{n}$ basis states, which is formulated as:

$$
|\psi\rangle=\sum_{i=0}^{2^{n}-1} \alpha_{i}|i\rangle,
$$

with $\alpha_{i} \in \mathbb{C}: \sum_{i=0}^{2^{n}-1}\left|\alpha_{i}\right|^{2}=1$.

Hence, while $n$ classical bits are only ever in one of the $2^{n}$ possible states at any given moment, an $n$-qubit register can be in a superposition of all of the possible states.

Quantum algorithms manipulate the amplitude and phase of either the total [31] or the partial [32] quantum wave function of the system to build interference patterns, affecting the probability of measuring particular values to execute the algorithms mentioned above

${ }^{8}$ Let us consider the following concrete examples to clarify this important concept. Interference may be readily visualized with the aid of two consecutive applications of the Hadamard gate. The first application creates a superposition, $H|0\rangle=\frac{|0\rangle+|1\rangle}{\sqrt{2}}=|+\rangle$, while the second application of a Hadamard gives

$$
H|+\rangle=\frac{H|0\rangle+H|1\rangle}{\sqrt{2}}=\frac{|0\rangle+|1\rangle+|0\rangle-|1\rangle}{2}=|0\rangle,
$$

in which we see constructive interference strengthening the $|0\rangle$ state and destructive interference canceling out the $|1\rangle$ state. This effect is at the heart of the most general quantum algorithm, amplitude amplification, exemplified in Grover's algorithm [17]. For example, let us begin with a 2-qubit system in the uniform superposition state $|\psi\rangle=\frac{1}{2}(|00\rangle+|01\rangle+|10\rangle+|11\rangle)$. Let us assume that the state $|01\rangle$ represents the correct answer we are looking for Initially, the probability of obtaining $|01\rangle$ on measurement is $\left|\frac{1}{2}\right|^{2}=1 / 4$. Let us now apply a unitary transformation known as the oracle to $|\psi\rangle$ to change the sign of $|01\rangle$, equivalent to a shift in the phase of $\pi, e^{i \pi}=-1$. We then obtain the new state $\left|\psi^{\prime}\right\rangle=\frac{1}{2}(|00\rangle-|01\rangle+|10\rangle+|11\rangle)$. The probability of obtaining $|01\rangle$ is not changed and is still $1 / 4$. Next, the diffusion operator $A=2|\psi\rangle\langle\psi|-I$ (for details about quantum transformation, please refer to Sec. III-F creates quantum interference, resulting in the state $\left|\psi^{\prime \prime}\right\rangle=$ $A\left|\psi^{\prime}\right\rangle=|01\rangle$, and the probability of measuring the state $|01\rangle$ has been amplified to 1 . 
Recall that in Sec. II-B we have illustrated the measurement postulate for single qubit systems by emphasizing that the measurement outcome is probabilistic and the measurement transforms the quantum state into a state compatible with the measuring device. Similar statements hold also for measurements of multiple-qubit systems. However, in this case, the set of legitimate measurements and outcomes is significantly richer than in the single-qubit case [18]. In particular, in (6), $\left\{\left|\alpha_{i}\right|^{2}\right\}$ represent the probabilities of observing the basis vectors by measuring in such a basis. Providing a detailed treatment of the measurement of multiple-qubit systems is beyond the scope of this manuscript and we refer the readers who would like to explore further to [17], [18].

\section{E. Entanglement}

The vast majority of $n$-qubit states cannot be written as the tensor product of $n$ single-qubit states, even though they are all linear combinations of the basis states of the composite $n$-qubit system. Such states are termed entangled states [17], [18].

More formally, given a state $|\psi\rangle$ of a composite quantum system associated with the vector space $V$ and a tensor decomposition of $V$, namely $V=V_{0} \otimes V_{1} \otimes \ldots \otimes V_{n-1}$, the state $|\psi\rangle$ is separable, or unentangled - with respect to that decomposition - if it can be written as $|\psi\rangle=\left|v_{0}\right\rangle \otimes\left|v_{1}\right\rangle \otimes \ldots \otimes\left|v_{n-1}\right\rangle$, where $\left|v_{i}\right\rangle$ belongs to $V_{i}$. Otherwise, $|\psi\rangle$ is entangled with respect to this particular decomposition, but may be unentangled with other decompositions into subsystems 9 .

Let us consider for example the Bell states, called also EPR pairs 10 in honor of an article written by Einstein, Podolsky, and Rosen in 1935 [34]:

$$
\begin{aligned}
\left|\Phi^{+}\right\rangle & =\frac{1}{\sqrt{2}}(|00\rangle+|11\rangle) \\
\left|\Phi^{-}\right\rangle & =\frac{1}{\sqrt{2}}(|00\rangle-|11\rangle) \\
\left|\Psi^{+}\right\rangle & =\frac{1}{\sqrt{2}}(|01\rangle+|10\rangle) \\
\left|\Psi^{-}\right\rangle & =\frac{1}{\sqrt{2}}(|01\rangle-|10\rangle),
\end{aligned}
$$

\footnotetext{
${ }^{9}$ The entanglement is not an absolute property of a quantum state, but it depends on the particular decomposition of the composite system into subsystems under consideration. States entangled with respect to the singlequbit decomposition may be unentangled with respect to other decompositions into subsystems. Hence it must be specified or clear from the specific context which of the many legitimate tensor decompositions of $V$ is under consideration [18]. For instance [18], the four-qubit state $|\psi\rangle=$ $\frac{1}{2}\left(\left|0_{1} 0_{2} 0_{3} 0_{4}\right\rangle+\left|0_{1} 1_{2} 0_{3} 1_{4}\right\rangle+\left|1_{1} 0_{2} 1_{3} 04\right\rangle+\left|1_{1} 1_{2} 1_{3} 1_{4}\right\rangle\right)-$ where the subscripts denote the different qubits - is entangled with respect to the decomposition into single qubits, since it cannot be expressed as the tensor product of four single-qubit states. However, $|\psi\rangle$ is not entangled with respect to the system decomposition of a subsystem consisting of the first and third qubit and a subsystem consisting of the second and fourth qubit, since it can be re-written as the tensor product of two-qubit states $|\psi\rangle=\frac{1}{\sqrt{2}}\left(\left|0_{1} 0_{3}\right\rangle+\left|1_{1} 1_{3}\right\rangle\right) \otimes \frac{1}{\sqrt{2}}\left(\left|0_{2} 0_{4}\right\rangle+\left|1_{2} 1_{4}\right\rangle\right)$.

${ }^{10}$ We note that the original EPR entangled states are not of the form reported in equations [8]-[11, which was introduced by D. Bohm [33].
}

which represents four maximally entangled 2-qubit states. The Bell states cannot be decomposed, since it is impossible to find $a_{0}, a_{1}, b_{0}, b_{1}$ for assuring that:

$$
\left(a_{0}|0\rangle+b_{0}|1\rangle\right) \otimes\left(a_{1}|0\rangle+b_{1}|1\rangle\right)=\left|\Phi^{ \pm}\right\rangle
$$

or equivalently, that:

$$
\left(a_{0}|0\rangle+b_{0}|1\rangle\right) \otimes\left(a_{1}|0\rangle+b_{1}|1\rangle\right)=\left|\Psi^{ \pm}\right\rangle .
$$

To better understand entanglement, let us consider, for example, $\left|\Phi^{+}\right\rangle$. By measuring each of the two qubits forming the EPR pair independently, one obtains a random distribution of zero and one outcomes with equal probability. However, if the results of the two independent measurements are compared, we find that every time the measurement of a qubit yielded zero so did the measurement of the other qubit, and the same happened with the outcome one. Indeed, according to quantum mechanics, as soon as one of the two qubits is measured, the state of the other also becomes instantaneously determined. So far, this behavior could be emulated via prior (classical) agreement between the parties holding the two qubits that are purportedly entangled. However, if the two parties independently, simultaneously, randomly choose their measurement bases ( $\mathrm{Z}, \mathrm{X}$, diagonal, or anti-diagonal), we can calculate a statistical measure of the correlation of their outcomes that exceeds anything achievable classically unless the parties can communicate instantaneously. This quantum entanglement behavior led Einstein and his colleagues to the so-called EPR paradox: the measurement of a qubit instantaneously changes the state of the second qubit, regardless of the distance separating the two qubits. Einstein, Podolsky and Rosen provided a qualitative thought experiment that originally raised this concern; Bell showed that it could be tested statistically (entanglement of a single state can never be proven; it is always a statistical test of a large number of similarly-created states). At the time of writing the most common form of this test is constituted by the CHSH inequality [35], [36]. This phenomenon seems to suggest that information is being transmitted faster than light, violating the theory of relativity. But the paradox is illusory, since entanglement does not allow the transmission of information faster than light, as it will be clarified in Sec. III.

\section{F. Quantum State Transformations}

Closed quantum systems evolve in time according to deterministic, reversible unitary operations [5], [17], [18], [37]. Hence Nature does not allow arbitrary transformations of a closed quantum system. That is, the state $|\psi(t)\rangle$ of the system at time $t$ is related to the state $|\psi(0)\rangle$ of the system at an initial time instant 0 through a unitary operator $U(\cdot)$, which depends only on the time instants $t$ and 0 :

$$
|\psi(t)\rangle=U(|\psi(0)\rangle)
$$

Remark 1. Any linear operator $A: V \rightarrow W$ between vector spaces $V$ and $W$ admits a matrix representation that is completely equivalent to the operator $A(\cdot)$. Hence, the matrix representation and the operator are interchangeable. 
Consequently, in the following, we will use the same symbol to denote both of them without any loss of generality.

Remark 2. Unitary operators are special linear operators having unitary matrix representations, i.e. we have $U^{\dagger} U=I$. A unitary operator is an invertible operator satisfying $U^{-1}=U^{\dagger}$ [38].

The unitary operators (or equivalently the quantum transformations) can be regarded as gates in the circuit model usually adopted both in quantum computation and in quantum information processing. Hence, although experimentalists usually describe the behavior of a quantum system by emphasizing the temporal nature of the evolution, in the circuit model perspective the temporal dependence is hidden within inputoutput relationships (e.g., (14) and it can be re-written as $\left.|\psi\rangle_{\text {out }}=U\left(|\psi\rangle_{\text {in }}\right)\right)$ [5], [17], [18]. As a consequence, unless explicitly stated, in the following we will not emphasize the temporal dependence of a quantum gate.

Please note that the expressions "quantum transformation" or "quantum operator" refer to unitary operators applied to the state space, not to measurement operators. Geometrically speaking, all quantum state transformations may also be interpreted as rotations of the complex vector space associated with the quantum state space.

In Table I], we summarize the most popular quantum gates. Naturally, the Identity operation $I$ leaves the quantum state unchanged. The Pauli-X operation imposes a bit-flip, the Pauli-Z a phase-flip, while Pauli-Y represents a joint bit- and phase-flip. The Hadamard operation maps the basis states into superpositions. Finally, the Controlled-Not (CNOT) operation performs the Pauli-X operation on the second qubit whenever the first qubit is $|1\rangle$, and otherwise leaves it unchanged. It is important to further highlight that the Pauli-Z gate reported in Table $\mathrm{I}$ changes the relative phase of a superposition in the standard basis, and hence it is critical to quantum computation, as mentioned in Sec. II-C

An important consequence of the unitary nature of the quantum transformations is the no-cloning theorem [39]-[41]: unknown quantum states cannot be copied or cloned [17], [18]. Indeed, the no-cloning theorem has a deep and complex impact on the design of quantum communications, as it will be discussed in detail in the following sections. The corruption of the transmitted classical information by the noise does not imply the total loss of the information, since a copy of the original information can be stored at the source. By contrast, the corruption of the transmitted quantum information by decoherence implies the irreversible loss of information.

\section{G. Pure and Mixed States: The Density Matrix and Quantum Fidelity}

Quantum states can be either pure or mixed. So far, we have discussed only pure states. Briefly, a pure state is a quantum state that can be described by a ket vector, i.e. it can be written in the state-vector form. This does not mean that the statevector form has only one term: both $|0\rangle$ and $\alpha|0\rangle+\beta|1\rangle$ are pure states.

In contrast, mixed states are not viewed as true quantum states, but rather as a way of describing a system whose state is not fully defined. Instead, a mixed state is a probabilistic mixture of well-defined pure states, known as a statistical ensemble [5], [17], [18]. In particular, pure states give deterministic results when measured in appropriate bases, whereas mixed states give probabilistic results in all bases [18].

The individual qubits of an EPR pair constitute examples of mixed states, since they cannot be described individually by a well-defined ket vector. However, not all the mixed states are entangled. Becoming familiar with mixed states is important also because, as detailed in Sec. $\mathrm{V}$, the effect of decoherence is to transform pure states into mixed states.

Although there exist different interpretations of the mixed states [42]-[44], they are modeled in mathematical terms by density operators (or density matrices) [44], [45], regardless of the underlying physical interpretation. Their mean can be used for the rudimentary characterization of the statistical properties of an ensemble of quantum states. More precisely, let us assume that a quantum system is in one of a number of legitimate states $\left|\psi_{i}\right\rangle$, where $i$ is the state index, and the legitimate states have the respective probabilities $\left\{p_{i}\right\}$. In this context, $\left\{p_{i},\left|\psi_{i}\right\rangle\right\}$ is an ensemble of pure states. The density operator (or density matrix) $\rho$ of the system is defined as [17], [18]:

$$
\rho=\sum_{i} p_{i}\left|\psi_{i}\right\rangle\left\langle\psi_{i}\right|
$$

The density operator $\rho$ is a positive operator (and hence Hermitian) with trace one, $\operatorname{Tr}(\rho)=1$. For a pure state $|\psi\rangle$,

TABLE I: Popular Quantum Gates

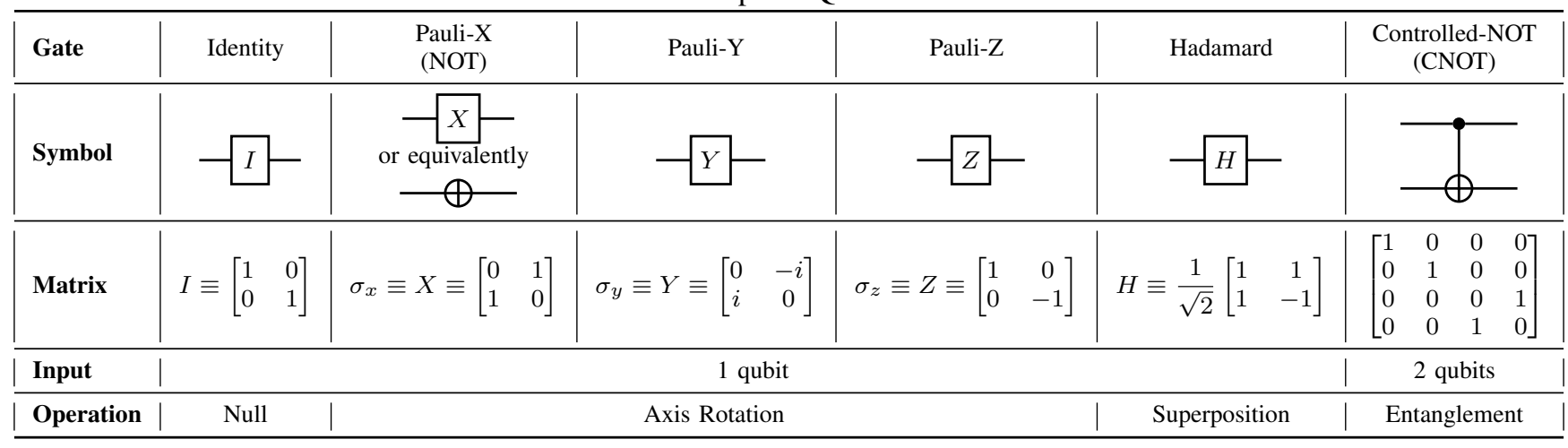




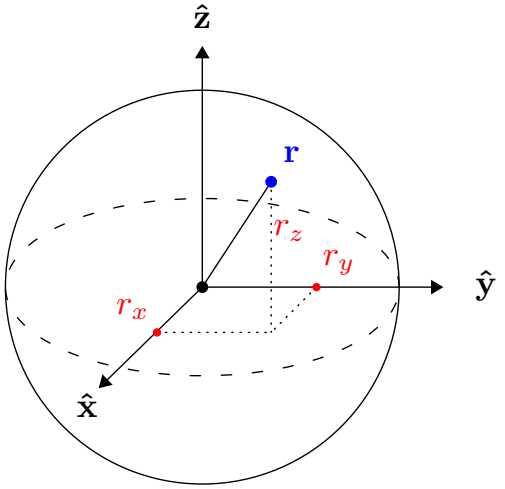

Fig. 2: Bloch Vector: geometrical representation of any (pure or mixed) quantum state in Cartesian coordinates $\left[r_{x}, r_{y}, r_{z}\right]$, which may be contrasted to its counterpart relaying on the spherical coordinates in Fig. 1

the density matrix is equal to $\rho=|\psi\rangle\langle\psi|$ with $\operatorname{Tr}\left(\rho^{2}\right)=1$, whereas for a mixed state $\operatorname{Tr}\left(\rho^{2}\right)<1$.

The imperfection of mixed states can be quantified by a fundamental figure of merit known as quantum fidelity. The fidelity $F$ of a mixed state associated with the density matrix $\rho$, with respect to a certain desired pure state $|\psi\rangle$, is a metric - taking values between 0 and 1 - of the distinguishability of the two quantum states, defined as [46]:

$$
F=\langle\psi|\rho| \psi\rangle \text {. }
$$

Based on this definition, the fidelity can be conceptually described as the "overlap" of the mixed state with the desired state $|\psi\rangle$. The fidelity is 1 for a pure state and it decreases as the decoherence degrades the "quality" of the state.

Finally, we note that the postulates of quantum mechanics can be reformulated in terms of the density operator [17]. This reformulation ${ }^{11}$ is mathematically equivalent to the description in terms of the state vector. Nevertheless, as mentioned before, the density operator approach is extensively utilized for characterizing the quantum imperfections, as discussed in Sec. V

\section{H. The Bloch Vector}

Geometrically, the Bloch sphere of Fig. 1 includes singlequbit mixed states. In fact, mixed states are constituted by linear combinations of pure states having non-negative weighting coefficients that sum to 1 . Hence it is not surprising that single-qubit mixed states can be viewed as laying within the interior of the Bloch sphere [18]. The precise connection with the geometry relies on the fact that any density matrix of a single-qubit system, which is a $2 \times 2$ matrix, can be written as [17], [18]:

$$
\rho=\left[\begin{array}{ll}
\rho^{00} & \rho^{01} \\
\rho^{10} & \rho^{11}
\end{array}\right]=\frac{1}{2}\left(I+r_{x} \sigma_{x}+r_{y} \sigma_{y}+r_{z} \sigma_{z}\right),
$$

\footnotetext{
${ }^{11}$ As an example, the postulate related to the evolution of a closed quantum system and reported in (14), can be reformulated by stating that the state $\rho$ of the system at time instant $t_{1}$ is related to the state $\rho^{\prime}$ of the system at time instant $t_{0}$ through a unitary operator $U$, which depends only on the time instants $t_{1}$ and $t_{0}: \rho=U \rho^{\prime} U^{\dagger}$.
}

where $\sigma_{x}, \sigma_{y}, \sigma_{z}$ represent the Pauli matrices defined in Tab. I and $r_{x}, r_{y}, r_{z}$ are the Cartesian coordinates of the quantum state considered [18], as shown in Fig. 2 Recall that its spherical coordinate based counterpart was shown in Fig. 1.

Hence, there exists a one-to-one mapping between any (pure or mixed) quantum state associated with the density matrix $\rho$ and the real three-dimensional vector $\mathbf{r}=\left[r_{x}, r_{y}, r_{z}\right] \in \mathcal{R}^{3}$, known as Bloch vector, where we have:

$$
\begin{aligned}
& r_{x}=\rho^{01}+\rho^{10}=2 \operatorname{Re}\left(\rho^{01}\right) \\
& r_{y}=i\left(\rho^{01}-\rho^{10}\right)=2 \operatorname{Im}\left(\rho^{10}\right) \\
& r_{z}=\rho^{00}-\rho^{11},
\end{aligned}
$$

with the norm of $\mathbf{r}$ being strictly smaller than one for mixed states, i.e. $\|\mathbf{r}\|<1$, while the norm being equal to one for pure states, i.e. $\|\mathbf{r}\|=1$. A similar relationship exists between a density matrix and the Cartesian coordinates, when multiplequbit systems are considered.

Indeed, the one-to-one mapping of $(18)$ between the density matrix $\rho$ and the Bloch vector $\mathbf{r}$ insightfully visualizes the effects of the 1-qubit quantum gates of Table II Specifically, commencing from (18) and then accounting for the evolution of quantum systems in terms of their density matrices formulated as $\rho_{\text {out }}=U \rho_{\text {in }} U^{\dagger}$, we arrive at:

$$
\mathbf{r}_{\text {in }}=\left[r_{x}, r_{y}, r_{z}\right] \stackrel{U}{\rightarrow}\left\{\begin{array}{ll}
\mathbf{r}_{\mathrm{out}}=\left[r_{x}, r_{y}, r_{z}\right] & \text { if } U=I \\
\mathbf{r}_{\mathrm{out}}=\left[r_{x},-r_{y},-r_{z}\right] & \text { if } U=X \\
\mathbf{r}_{\mathrm{out}}=\left[-r_{x}, r_{y},-r_{z}\right] & \text { if } U=Y . \\
\mathbf{r}_{\mathrm{out}}=\left[-r_{x},-r_{y}, r_{z}\right] & \text { if } U=Z \\
\mathbf{r}_{\mathrm{out}}=\left[r_{z},-r_{y}, r_{x}\right] & \text { if } U=H
\end{array} .\right.
$$

Remark 3. It is important to underline that - despite the luring illusion that the Pauli-X gate affects in some way the $\mathrm{X}$-coordinate of the Bloch vector - the Pauli-X gate leaves the $\mathrm{x}$-coordinate unchanged but it affects both the $\mathrm{y}$ - and the Z-coordinate. Similar considerations hold for the Pauli-Y and Pauli-Z gates, since they leave the $\mathrm{y}$ - and the $\mathrm{Z}$-coordinate unaltered, respectively, while affecting the remaining two coordinates.

\section{From TRANSMisSiON TO TELEPORTATION}

\section{A. Quantum Teleportation Overview}

Let us assume that a quantum state $|\psi\rangle$ must be transmitted from a sender, say Alice, to a remote receiver, say Bob.

We assume without any loss of generality that $|\psi\rangle$ is a pure qubit, yielding:

$$
|\psi\rangle=\alpha|0\rangle+\beta|1\rangle .
$$

If the transmitter knows the quantum state $|\psi\rangle$, i.e., if Alice knows $\alpha$ and $\beta$, the task can be accomplished by transmitting the values of $\alpha$ and $\beta$ to Bob and by letting Bob prepare $|\psi\rangle$, i.e., to "transform" a default state into $|\psi\rangle$ with the aid of Bob's local operations. Indeed, several open questions arise in conjunction with practical quantum state preparation [47]-[49], such as the specific construction of a set of universal gates, the required depth of the quantum circuit, or the minimum required fidelity of the reconstructed state. Nevertheless, from a communications engineering perspective, 


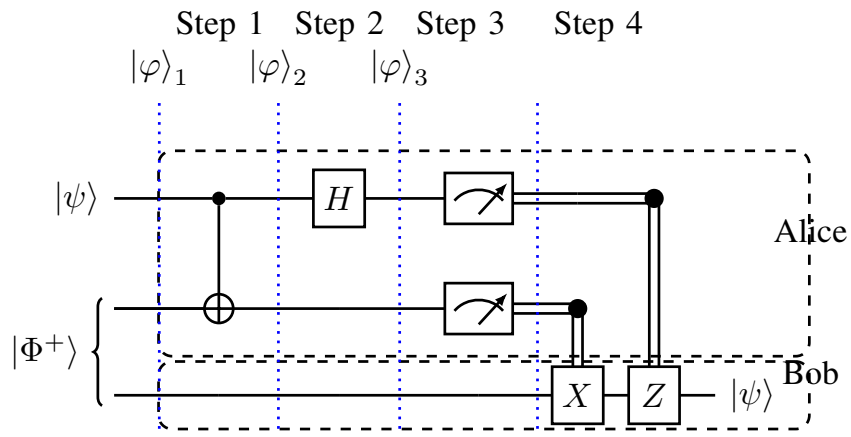

Fig. 3: Quantum Teleportation Circuit, where $|\psi\rangle$ denotes the unknown state to be transmitted from Alice to Bob, while $\left|\Phi^{+}\right\rangle$denotes the EPR pair generated and distributed so that one qubit is stored at Alice and another qubit is stored at Bob. Furthermore, $|\varphi\rangle_{i}$ denotes the global quantum state at the $i$-th step. The symbol $\rightarrow$ denotes the measurement operation and the double-line $=$ represents the transmission of a classical bit from Alice to Bob.

the communication task can be accomplished - at least in principle the aid of classical communication resources.

However, in the most general case $\mathrm{e}^{12}$ the transmitter does not know the quantum state $|\psi\rangle$, and the task cannot be accomplished with the aid of pure classical communication resources. In fact, the quantum measurement postulate prevents Alice from assessing $\alpha$ and $\beta$ with the aid of a quantum measurement, which would irreversibly alter the original quantum state. Furthermore, the no-cloning theorem prevents Alice from preparing multiple copies of $|\psi\rangle$ and estimating $\alpha, \beta$ by simply measuring the copies.

In other words, quantum mechanics does not allow a qubit to be copied or measured. Hence, although a photon is capable of conveying a qubit and it can be directly transmitted to a remote node - e.g., via a fiber link - if the traveling photon is lost due to attenuation or corrupted by decoherence, the original quantum information cannot be recovered via a measuring process or by re-transmitting a copy of the qubit. As a consequence, we cannot directly borrow techniques from classical communications. With the impressive exception of the Chinese satellite experiment, direct transmission of qubits has only been demonstrated over relatively short distances either via optical fibers or free space - for rather specific applications that can tolerate low transmission success rates, as exemplified by Quantum Key Distribution (QKD) and Quantum Secure Direct Communication (QSDC) networks [11], [19].

Thankfully, Quantum Teleportation ${ }^{13}$ constitutes a priceless technique of transmitting qubits without the physical transfer

\footnotetext{
${ }^{12}$ This case arises, for instance, in distributed quantum computing. Indeed, the quantum state obtained at a certain computing step of a distributed quantum algorithm is unknown by definition. Furthermore, any observation of the state before the conclusion of the quantum algorithm would imply an irreversible loss of information due to the quantum measurement postulate.

${ }^{13}$ Although we focus our attention on discrete-variable quantum teleportation, continuous-variable quantum teleportation is possible [50], [51], and we refer the reader to $[52]$ for an in-depth discussion.
}

of the particle storing the qubit. As shown in Figure 3, with the aid of local operations and an EPR pair ${ }^{14}$ shared between the source and destination, quantum teleportation allows us to "transmit" an unknown quantum state [1], [3], [5], [17], as will be detailed in the next section.

Quantum teleportation implies the destruction of both the original qubit (encoding the quantum information to be transmitted) and the EPR member at the source, as a consequence of a measurement. Indeed, as will be shown in Sec. III-B. the original qubit is reconstructed at the destination, once the output of the measurement at the source -2 classical bits is received through a finite-delay classical link, obeying the speed of light in an optical link for example.

\section{B. Quantum Teleportation: Mathematical Details}

In a nutshell, the teleportation process of Fig. 3 takes as its input the state $|\psi\rangle$ to be teleported and an EPR pair shared between Alice and Bob. Each of the four states $\left|\Phi^{ \pm}\right\rangle,\left|\Psi^{ \pm}\right\rangle$ can be used for quantum teleportation, given that the state is fixed in advance by mutual agreement between Alice as well as Bob, and this mutual agreement can be achieved with the aid of a finite-delay classical link.

In the following, we assume without loss of generality that Alice and Bob share the state $\left|\Phi^{+}\right\rangle=(|00\rangle+|11\rangle) / \sqrt{2}$, as shown in Fig. 3 Hence, the initial global state $\left|\varphi_{1}\right\rangle=|\psi\rangle \otimes$ $\left|\Phi^{+}\right\rangle$depicted in Fig. 3 is:

$$
\left|\varphi_{1}\right\rangle=\alpha|0\rangle \otimes \frac{|00\rangle+|11\rangle}{\sqrt{2}}+\beta|1\rangle \otimes \frac{|00\rangle+|11\rangle}{\sqrt{2}} .
$$

By following the convention that the pair of leftmost qubits belongs to Alice and the rightmost qubit belongs to Bob, 21 becomes equivalent to:

$$
\left|\varphi_{1}\right\rangle=(\alpha|000\rangle+\alpha|011\rangle+\beta|100\rangle+\beta|111\rangle) / \sqrt{2} .
$$

Step 1. The teleportation process of Fig. 3 starts with Alice applying the CNOT gate of Table $\mathrm{I}$ to the pair of qubits at her side. By recalling that the CNOT gate maps state $|10\rangle$ into $|11\rangle$ and vice versa, the global state $\left|\varphi_{2}\right\rangle$ after the CNOT gate (Step 1 in Fig. 3) becomes:

$$
\left|\varphi_{2}\right\rangle=(\alpha|000\rangle+\alpha|011\rangle+\beta|110\rangle+\beta|101\rangle) / \sqrt{2} \text {. }
$$

Step 2. Then, as seen in Fig. 3, Alice applies the $\mathrm{H}$ gate of Table I to the qubit to be teleported, i.e. to the leftmost qubit in 23 . By recalling that the $H$ gate maps $|0\rangle$ into $\frac{|0\rangle+|1\rangle}{\sqrt{2}}$ and $|1\rangle$ into $\frac{|0\rangle-|1\rangle}{\sqrt{2}}$, the global state $\left|\varphi_{3}\right\rangle$ after the $H$ gate (Step 2 in Fig. 3) is obtained from (23) in the following form:

$$
\begin{aligned}
&\left|\varphi_{3}\right\rangle=( \alpha|000\rangle+\alpha|100\rangle+\alpha|011\rangle+\alpha|111\rangle+ \\
&\beta|010\rangle-\beta|110\rangle+\beta|001\rangle-\beta|101\rangle) / 2 .
\end{aligned}
$$

By gathering the two leftmost qubits belonging to Alice, 24 becomes equivalent to:

$$
\begin{aligned}
\left|\varphi_{3}\right\rangle=(|00\rangle \otimes(\alpha|0\rangle+\beta|1\rangle)+|01\rangle \otimes(\alpha|1\rangle+\beta|0\rangle)+ \\
|10\rangle \otimes(\alpha|0\rangle-\beta|1\rangle)+|11\rangle \otimes(\alpha|1\rangle-\beta|0\rangle)) / 2
\end{aligned}
$$

\footnotetext{
${ }^{14}$ Although multipartite-entangled states can be used for quantum teleportation, here we restrict our attention to EPR pairs for the sake of simplicity.
} 
Step 3. Then, as indicated in Fig. 3. Alice jointly measures the pair of qubits at her side. Remarkably, and regardless of the values of $\alpha$ and $\beta$, Alice has a $25 \%$ chance of finding each of the four combinations $|00\rangle,|01\rangle,|10\rangle$ and $|11\rangle$. Alice's measurement operation instantaneously fixes Bob's qubit, regardless of the distance between Alice and Bob as a consequence of the entanglement described in Sec. II-B $\left.\right|^{5}$ However, Bob can only recover the original qubit $|\psi\rangle$ after he correctly receives the pair of classical bits conveying the specific results of Alice's measurement. Naturally, this classical transmission has to obey the speed of light.

Step 4. Specifically, four terms can be identified in 25, depending on the particular state of the two qubits at Alice. When Alice's qubits are in state $|00\rangle$, then Bob's qubit is in the state $\alpha|0\rangle+\beta|1\rangle$, i.e. Bob's qubit is identical to the original quantum state $|\psi\rangle$. Hence, if Alice obtains 00 by jointly measuring her pair of qubits and she communicates the outcome to Bob, then Bob can directly recover the original quantum state $|\psi\rangle$ from the qubit at his side, without any quantum-domain operation. At this step, we say that the original quantum state $|\psi\rangle$ has been teleported to Bob's side.

Alternatively, if Alice's qubits are in the state $|10\rangle$, then Bob's qubit is in the state $\alpha|0\rangle-\beta|1\rangle$. Hence, if Alice obtains 10 by jointly measuring her pair of qubits, once Bob receives the measurement outcome via a classical link, he can recover the original quantum state $|\psi\rangle$ by applying the $\mathrm{Z}$ gate of Table $\mathrm{I}$ (that maps $|0\rangle$ in $|0\rangle$ and $|1\rangle$ in $-|1\rangle$ ) to the qubit at his side, as seen in Fig. 3. Again, at this step, we say that the original quantum state $|\psi\rangle$ has been teleported to Bob's side. Similarly [17], if the measurement at Alice's side is 01 or 11 , Bob recovers $|\psi\rangle$ by simply applying either the $\mathrm{X}$ gate of Table I] or the $\mathrm{X}$ gate followed by the $\mathrm{Z}$ gate to the qubit at his side.

Remark 4. Based on the above discussions, it becomes clear that the teleportation process of a single qubit requires: i) the generation and the distribution of an EPR pair between the source and destination, ii) a finite-delay classical communication channel for conveying the pair of classical bits resulting from the measurement. Hence, it is worthwhile noting that having a tight integration between the pair of classical and quantum resources is necessary in a quantum network [1]. Regarding the EPR pair, the measurement at the source destroys the entanglement. Hence, if another qubit has to be teleported, a new EPR pair must be created as well as distributed between the source and destination.

\section{Practical Entanglement Generation and Distribution}

As pointed out above, the entanglement generation and distribution functionality is the key ingredient of quantum teleportation. Here, as a preliminary to the modeling to be discussed in Sec. IV, we briefly review this functionality from a practical perspective.

In a nutshell, here we gloss over many of the details, but the generation of quantum entanglement requires that two (or

\footnotetext{
${ }^{15}$ Einstein referred to this phenomenon by the light-hearted parlance of a "spooky action at a distance".
}

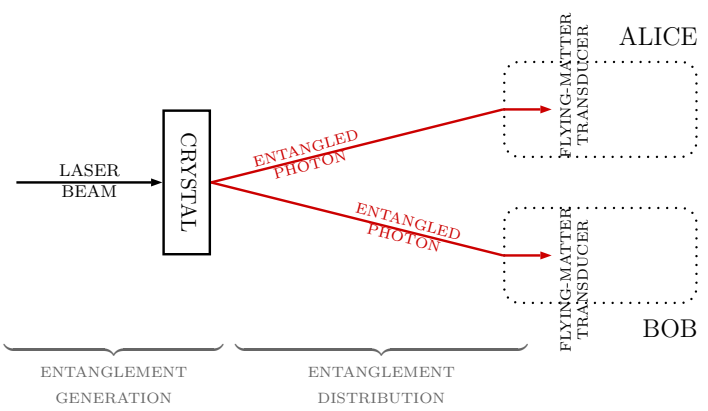

(a) Spontaneous Parametric Down-Conversion |53|: a laser beam is directed toward a non-linear crystal, which occasionally splits photon beams into pairs of polarization-entangled photons.

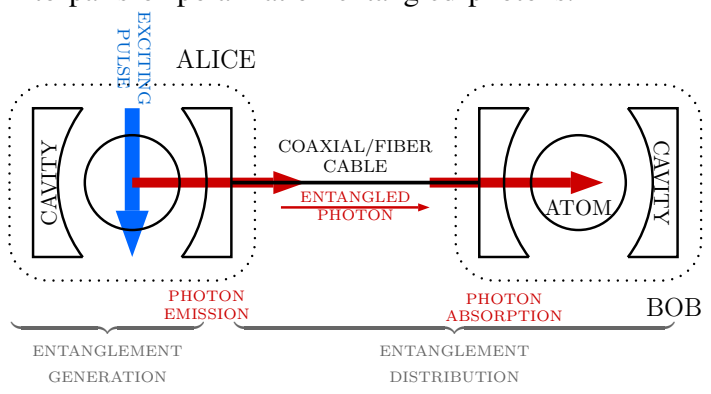

(b) An atom strongly coupled with an optical cavity is excited by a laser beam [54]-[57]. The resultant photon leaks out of the cavity, propagates as a wave packet through the cable, and enters an optical cavity at the second node, entangling the two remote atoms.

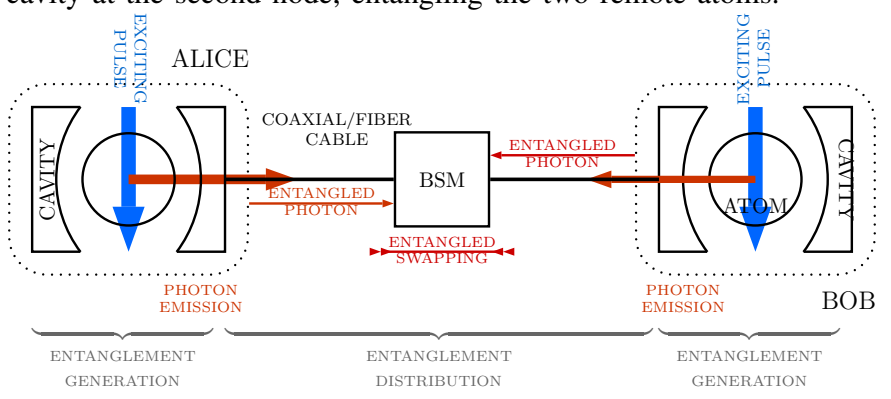

(c) Two atoms in optical cavities are simultaneously excited with a laser pulse [58]-[61], leading to the emission of two atom-entangled photons. By measuring the incoming photons, the Bell State Measurement (BSM) projects the atoms into an entangled quantum state.

Fig. 4: Practical schemes for entanglement generation and distribution. Regardless of the location of the entanglement generation functionality (at mid-point as in Fig. 4a, at source as in Fig. 4b, or at both end-points as in Fig. 4c [62]), a quantum link is needed to distribute the entanglement between Alice and Bob.

more) particles are in each other's spatial proximity - for example owing to their joint generation or due to their direct interaction - so that the state of any of these particles cannot be described independently from the state of the other.

Since Alice and Bob represent remote nodes, the entanglement generation occurring at one side must be complemented by the entanglement distribution functionality, which "moves" one of the entangled particles to the other side. In this context, there is a broad consensus in the community concerning the adoption of photons as the substrate for the so-called flying 


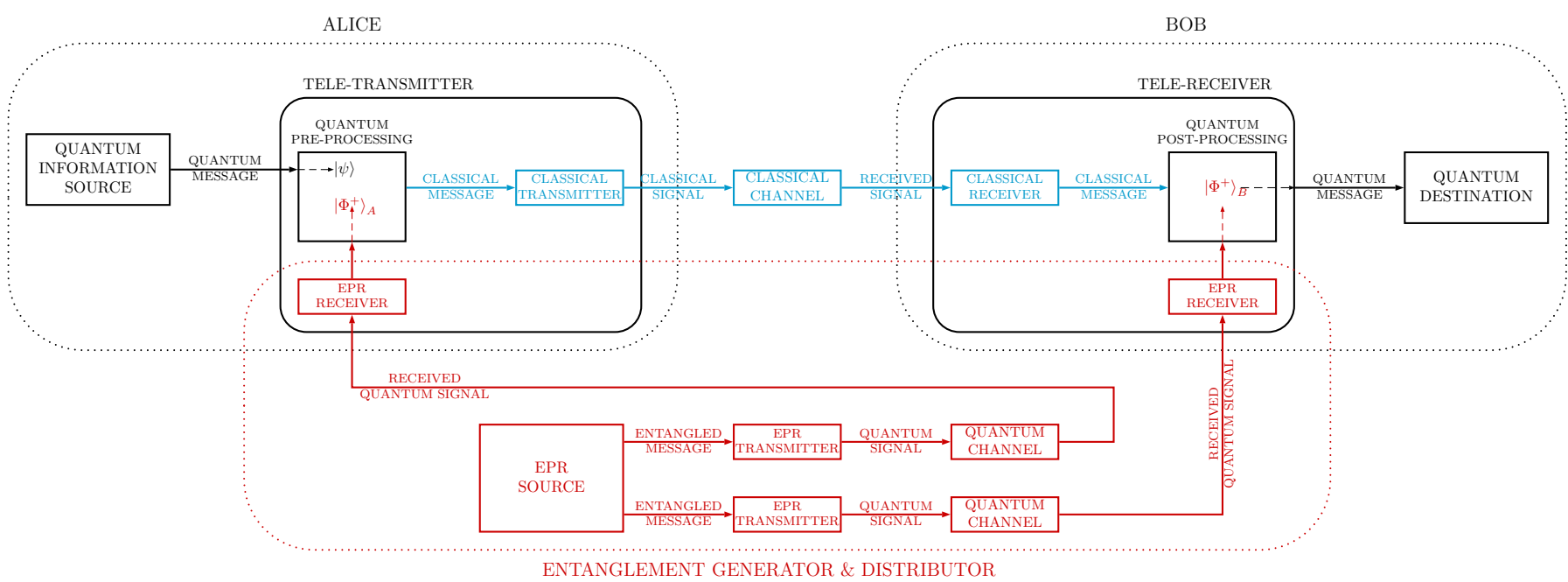

Fig. 5: A Noiseless Communication System Model of Quantum Teleportation.

qubits [59], i.e., as entanglement carriers. The rationale for this choice is related to the advantages provided by photons for entanglement distribution: moderate interaction with the environment leading to moderate decoherence as described in Sec. V] convenient control with standard optical components as well as high-speed low-loss transmissions.

Indeed, one of the basic schemes conceived for entanglement generation - namely, the Type-II spontaneous parametric down-conversion detailed in [53] - employs photons for both entanglement generation and distribution, as summarized in Fig. 4a By pointing a laser beam toward a non-linear crystal, two intersecting cones emerge from the crystal with a vertically polarized photon on the upper cone and a horizontally polarized photon on the lower cone. Polarizationentangled photons in one of the Bell states are generated at the two intersections of the cones. The entangled photons travel through a quantum channel to reach their destinations, namely Alice and Bob, where a transducer is invoked at each side [1] for "transferring" the entanglement from the flying qubit to the matter qubit, i.e. to a qubit suitable for information processing/storage within a quantum device [63]. Indeed, the transducer is needed at the destination, since the very feature that makes photons attractive for entanglement distribution - namely, their moderate interaction with the environment represents a major drawback when it comes to employing photons as substrate for quantum-domain processing/storage. In fact, quantum processing/storage requires the qubits to interact with each other [64]. We further observe that polarizationentangled photons at telecom wavelength can be obtained through the setup in [65], with advantages for long-distance quantum communications.

A different scheme designed for generating and distributing the entanglement is depicted in Fig. $4 \mathrm{~b}$ This scheme utilizes atoms in optical cavities [54]-[57] linked by a photonic channel, such as a coaxial/fiber cable. Specifically, the first step is to excite an atom coupled with an optical cavity by a laser pulse, which leads to the emission of a photon into the cavity mode. The polarization of the photon is entangled with some internal state of the atom. The photon, exiting the first cavity and traveling along the quantum channel, reaches the second cavity, where it is coherently absorbed and its polarization is mapped onto the state of the remote atom. The atom-photon entanglement is thus converted into entanglement between the two remote atoms. In this scheme, the cavity acts as the matterflying transducer described in the context of the first scheme.

Finally, a third scheme conceived for generating and distributing the entanglement is summarized in Fig. 4c Here, both the atoms are simultaneously excited with a laser pulse, leading to the emission of a photon in each cavity. Each photon is entangled with the emitting atom and travels along a quantum channel. Both the photons are then combined at a beam-splitter-based Bell State Measurement (BSM), which stochastically projects ${ }^{16}$ the remote atoms into an entangled quantum state [58]-[61]. This scheme has also been proposed in the context of Nitrogen-Vacancy (NV) defect centers in diamonds [68]. Finally, it has been extended to artificial atoms such as transmons [69], which constitute one of the most popular substrates for computational qubits. Similarly to the scheme in Fig. 4b, the cavity in this scheme also acts as a matter-flying transducer.

We further observe, with reference to the schemes in Figs. $4 \mathrm{~b}$ and $4 \mathrm{c}$, that they assume the excitation of single atomic particles. Alternatively, Duan et al. [70] proposed using a collective excitation in atomic ensembles, which allows simpler realization and improved generation efficiency.

Although the above discussion is far from being exhaustive ${ }^{17}$. some related considerations can be drawn as follows. The specific "location" of the entanglement generation varies among the schemes [62]. For example, in the scheme of Fig. 4a it is "at mid-point". By contrast, it is "at the source" in Fig. $4 \mathrm{~b}$ and "at both end-points" in Fig. 4c. Nevertheless,

\footnotetext{
${ }^{16}$ This procedure is also known under the name of entanglement swapping: the entanglement is swapped from the two original atom-photon pairs to the atom-atom pair [66], 67].

${ }^{17}$ Additionally, the entanglement can be distributed by literally moving stationary qubits and their associated hardware - after entangling them at one party. However, this scheme is far from being scalable.
} 
each scheme requires a quantum channel between Alice and Bob for entanglement distribution. Furthermore, a transducer is needed ${ }^{18}$ for interfacing the matter qubit with the flying qubit traveling through the quantum channel. These key features of practical entanglement generation/distribution are exploited in the next section for modeling quantum teleportation from a communications engineering perspective.

\section{A Noiseless Communication System Model of QUANTUM TELEPORTATION}

Based on the discussions developed in the previous section, it comes to light that a communication system model conceived for quantum teleportation relies on the tight integration of both classical/quantum operations and communications. Hence, the classical communication system model originally proposed by Shannon [26] must be revised to account for the specific nature of quantum teleportation. Hence we propose the model depicted in Fig. 5, where the different system blocks summarized in Table [II and discussed below may be readily identified. For the sake of clarity, we also highlight in Fig. 6 the communication functionalities of the aforementioned blocks with reference to the quantum teleportation circuit described in Fig. 3 .

(a) The Quantum Information Source of Fig. 5 provides the Tele-Transmitter with the unknown quantum message to

\footnotetext{
${ }^{18}$ This is the case, unless the flying qubits are also used for computing as in the case of photonic-based quantum processors [64], although this research is still in its infancy.
}

be teleported to Bob and maps it to the qubit state $|\psi\rangle$, which again cannot be read or copied 19

(b) The Tele-Transmitter of Fig. 5 processes the quantum message mapped to $|\psi\rangle$ to produce a classical signal suitable for transmission over a classical channel. This operation is accomplished by the following sub-blocks of Fig. 5. the Quantum Pre-Processing, the Classical Transmitter and the EPR Receiver. Specifically, the EPR Receiver is a sub-block of the Entanglement Generator \& Distributor super-block of Fig. 5, which supplies the Tele-Transmitter and the Tele-Receiver with the respective members of the entangled pair - e.g., $\left|\Phi^{+}\right\rangle_{A}$ and $\left|\Phi^{+}\right\rangle_{B}$. The Entanglement Generator \& Distributor as well as its sub-blocks are described in detail at the end of this section. The Quantum Pre-Processing jointly operates on $|\psi\rangle$ and $\left|\Phi^{+}\right\rangle_{A}$ by applying a sequence of operations, i.e., by performing a BSM, as detailed in Sec. III-B and further highlighted in Fig. 6. Explicitly, the CNOT gate of Table $\mathrm{I}$ is applied to both the qubits at Alice's side, followed by applying the $\mathrm{H}$ gate of Table II to the qubit to be teleported, further followed by a joint measurement applied to both qubits. Hence its output - namely the result of the measurement - is a classical message, which is in turn mapped by the Classical Transmitter to a classical signal suitable for

${ }^{19} \mathrm{As}$ it will be detailed in the next section, this implies that classical error correction techniques, based on parity or repetition techniques, cannot be employed in a quantum network [71].

TABLE II: Communication System Modeling: Classical Communications vs Quantum Teleportation

\begin{tabular}{|c|c|c|}
\hline Block & Classical Communication & Quantum Teleportation \\
\hline Information Source & $\begin{array}{l}\text { The source output can be: } \\
\text { - safely read without altering the embedded information } \\
\text { - copied, and hence it can be re-transmitted whenever } \\
\text { corrupted by noise }\end{array}$ & $\begin{array}{l}\text { The source output cannot be: } \\
\text { - read without altering the embedded information - the quantum } \\
\text { measurement postulate } \\
\text { - duplicated - the no-cloning theorem - and hence it cannot be } \\
\text { re-transmitted when corrupted by noise }\end{array}$ \\
\hline EPR Source & Absent & Entangling a certain inner state of two particles \\
\hline & $\begin{array}{l}\text { Classical Transmitter: } \\
\text { - mapping classical information into a classical signal } \\
\text { suitable for transmission over a classical channel }\end{array}$ & $\begin{array}{l}\text { Classical Transmitter } \\
\text { - mapping the (classical) output of the quantum pre-processing into } \\
\text { a classical signal suitable for the classical channel }\end{array}$ \\
\hline Transmitter & $\begin{array}{l}\text { EPR Transmitter: } \\
\text { • absent }\end{array}$ & $\begin{array}{l}\text { EPR Transmitter } \\
\text { - mapping the entangled particle into a quantum signal suitable for } \\
\text { the considered quantum channel, for conveying the entanglement } \\
\text { at the remote node }\end{array}$ \\
\hline \multirow{3}{*}{ Channel } & $\begin{array}{l}\text { Classical Channel } \\
\text { - the communication range can be extended through clas- } \\
\text { sical amplify-and-forward or decode-and-forward tech- } \\
\text { niques, since a classical signal can be measured without } \\
\text { altering the encoded information }\end{array}$ & $\begin{array}{l}\text { Classical Channel } \\
\text { - medium used to transmit the classical signal from the Tele- } \\
\text { Transmitter to the Tele-Receiver }\end{array}$ \\
\hline & $\begin{array}{c}\text { Quantum Channel } \\
\text { • absent }\end{array}$ & $\begin{array}{l}\text { Quantum Channel } \\
\text { - conveying the entanglement to remote nodes } \\
\text { - the communication range cannot be extended through classical } \\
\text { amplify-and-forward or decode-and-forward techniques, due to the } \\
\text { quantum measurement. Quantum Repeaters should be adopted }\end{array}$ \\
\hline & $\begin{array}{l}\text { Classical Receiver } \\
\text { - decoding the classical message from the received classi- } \\
\text { cal signal }\end{array}$ & $\begin{array}{l}\text { Classical Receiver } \\
\text { - decoding the classical input of the quantum post-processing block } \\
\text { from the received classical signal }\end{array}$ \\
\hline Receiver & $\begin{array}{c}\text { EPR Receiver } \\
\bullet \quad \text { absent }\end{array}$ & $\begin{array}{l}\text { EPR Receiver } \\
\text { - decoding the entangled input of the quantum pre-processing and } \\
\text { post-processing blocks from the received quantum signals }\end{array}$ \\
\hline
\end{tabular}




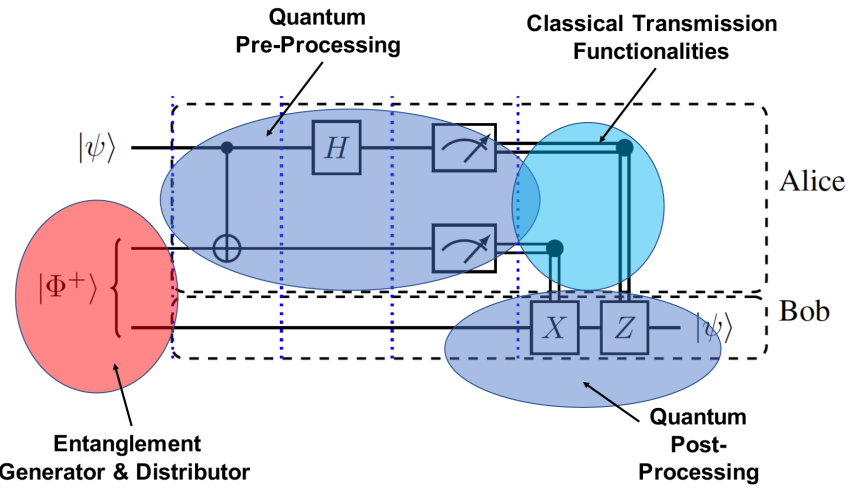

Fig. 6: Quantum Teleportation Circuit of Fig. 3 interpreted in the light of Fig. 5 .

transmission to Bob over a classical channel.

(c) The Tele-Receiver of Fig. 5 performs the inverse operations of the Tele-Transmitter: it reconstructs the unknown quantum message from the received classical signal. This operation is accomplished by the following subblocks of Fig. 5, the Classical Receiver, the Quantum Post-Processing and the EPR Receiver. Specifically, first the Classical Receiver decodes the received classical signal into a classical message. Then, the Quantum PostProcessing recovers $|\psi\rangle$ from $\left|\Phi^{+}\right\rangle_{B}$, which is provided by the EPR Receiver, with the sequence of quantum operations (gates) indicated by the classical message (see Sec. III-B as also highlighted in Fig. 6

(d) The Entanglement Generator \& Distributor of Fig. 5 is the super-block responsible for the generation and distribution of the EPR pair members $\left|\Phi^{+}\right\rangle_{A}$ and $\left|\Phi^{+}\right\rangle_{B}$ at Alice and Bob, respectively, as also highlighted in Fig. 6 Following from our discussion in Sec. [III-C, the Entanglement Generator \& Distributor super-block is constituted by four blocks, as shown in Fig. 5. Although the specific location and the physical implementation of each block may vary - depending on the particulars of the practical scheme adopted for entanglement generation and distribution - the model of Fig. 5 allows us to highlight each communication functionality required for generating and distributing the EPR pair to remote nodes.

(d1) The EPR Source of Fig. 5 generates the EPR pair by maximally entangling a certain inner state of two particles. The particular nature of the particles depends on the specific scheme considered - ranging from photons through atoms to artificial atoms in superconducting circuits. The location of the EPR source may vary as well, as shown in Figs. 4a. 4c. Nevertheless, by utilizing a terminology familiar to the communications engineering community the entangled states represent the "entangled messages" to be transmitted to both Alice and Bob.

(d2) The EPR Transmitter of Fig. 5 processes the entangled message to produce a quantum signal suitable for transmission over a quantum channel, such as an optical fiber or Free-Space Optical (FSO) link. Again, the broad consensus is that of adopting photons as substrate for flying qubits [72]. However, the entanglement can be mapped to a photon's different features - such as its polarization, time-bin, etc. [64]. Hence, these degrees of freedom are exploited by the EPR Transmitter to produce a quantum signal suitable for transmission over the specific quantum channel.

(d3) The Quantum Channel block of Fig. 5 represents the medium used for transmitting the quantum signal from the EPR Transmitter to the EPR Receiver. The quantum channel characteristics as well as the maximum achievable communication range vary significantly, depending on the specific choice of transmission medium - i.e., FSO terrestrial of satellite channel or alternatively optical fiber channel. Furthermore, in contrast to classical channels, where the communication range can be extended using classical amplify-and-forward or decode-andforward techniques, quantum channels require the adoption of quantum repeater 20 [74], since the quantum signal cannot be measured without irreversibly altering the original quantum signal due to the measurement postulate [5], [21], [62], [75].

(d4) The EPR Receiver of Fig. 5 performs the inverse operation of the EPR Transmitter, by extracting the entangled state from the received quantum signal. It is responsible for providing the Quantum PreProcessing and the Quantum Post-Processing with the entangled pair members $\left|\Phi^{+}\right\rangle_{A}$ and $\left|\Phi^{+}\right\rangle_{B}$.

Remark 5. Again, the modeling of the Entanglement Generator \& Distributor super-block of Fig. 5 aims for providing a general portrayal of the communication functionalities required. However, the physical-counterparts of its component blocks vary, depending on the specific choices of the entanglement generation/distribution technique adopted, as described in Sec. III-C. For instance, the schemes in Figs. $4 \mathrm{a} 4 \mathrm{~b}$ employ a single EPR Source, which is located either at Alice or halfway between Alice and Bob. By contrast, the scheme in Fig. 4c employs a pair of EPR sources, one at Alice and one at Bob. As regards to the EPR Transmitter, we have a single transmitter in Figs. 4a-4b (the crystal in the former, acting also as the EPR source, and the cavity in the latter, acting as the matter-flying qubit transducer) while two transmitters in Fig. 4c (the cavities at each side, acting as transducer as well). Finally, regarding the EPR Receiver, in Fig. 4a this functionality is performed by the matter-flying transducers located at Alice's side and at Bob's side, whereas there exists

\footnotetext{
${ }^{20}$ The BSM block of Fig. $4 \mathrm{c}$ may be regarded as a (very) basic quantum repeater [67]. Please refer to Sec. VII-B for further details about quantum repeaters $|73|$.
} 


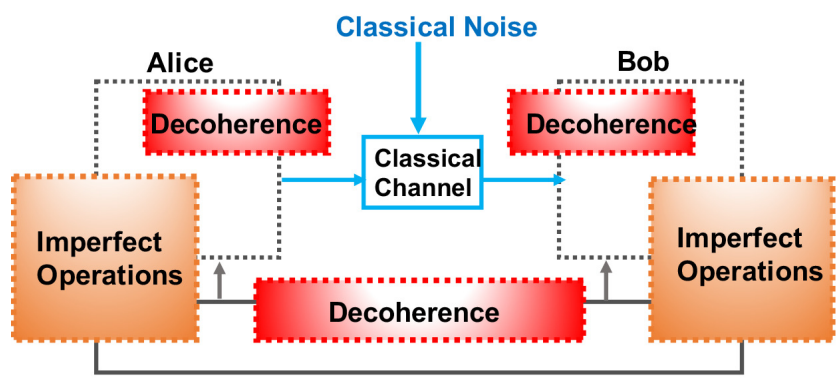

Entanglement Generator \& Distributor

Fig. 7: Imperfections Contaminating the Quantum Teleportation Process.

a unique physical EPR Receiver ${ }^{21}$ - the cavity - at Bob's side in Fig. 4b. By contrast, no physical EPR Receiver is present in Fig. $4 \mathrm{c}$. since the EPR pair members are locally generated at both Alice's and Bob's side through entanglement swapping. In other words, in Fig. 4c, the EPR Receiver block is "virtual" at both Alice's and Bob's sides, since its communication functionality, i.e., the process of "receiving" the member of the entangled pair is fulfilled without the physical reception of the particle, which is the phenomenon being exploited by the entanglement swapping.

Remark 6. It is worthwhile noting that in Fig. 5 the quantum equivalent of the classical source-encoder block is absent. Specifically, the classical source-encoder is responsible for efficiently representing the source output in a sequence of binary digits with little or no redundancy [76]. However, this functionality is based on the assumption that any classical information can be read anywhere at any time, whilst this does not hold for the quantum domain. Hence, a one-to-one mapping between the classical source-encoder and a quantumequivalent source-encoder may not be feasible. Hence further research is needed. By contrast, the classical channel-encoder block is responsible for imposing carefully controlled redundancy on the message for detecting and correcting (to some degree) the errors inflicted by the channel imperfections [76]. Its quantum equivalent will be discussed in Sec. VII

Remark 7. As said, the aim of Fig. 5 is to highlight the communication functionalities needed to implement the quantum teleportation. For this, the focus is on a point-to-point communication link. The role of quantum repeaters, mentioned in Tab. III to increase the entanglement communication range is discussed in Sec. VII

\section{IMPERFECT QUANTUM TELEPORTATION}

The discussions of the previous section are valid under the idealized simplifying hypothesis of experiencing no decoherence. However, similarly to classical communications,

\footnotetext{
${ }^{21}$ The EPR Receiver block is "virtual" for Alice, since the process of "receiving" Alice's member of the entangled pair is fulfilled without the physical reception of the particle, by exploiting the above-mentioned "spooky action at a distance" determined by the interaction between the photon and the cavity.
}

the quantum communication model should account for the presence of realistic imperfections.

Again, realistic quantum systems suffer from undesired interactions with the environment. Hence, they constitute open rather than closed physical systems [37]. These interactions with the environment irreversibly affect any quantum state by the process of decoherence [17], [18], [77]. Decoherence is unavoidable and it affects not only the unknown quantum state to be teleported, but also the entanglement generation and distribution process required for implementing the quantum teleportation.

However, decoherence is not the only source of imperfections [1]. In fact, the quantum teleportation relies on a sequence of operations applied to the quantum states, as detailed before. The contamination of these operations further aggravates the imperfections of the quantum teleportation.

In Fig. 7, we depict the relationship of different imperfection ${ }^{22}$ degrading the fidelity of the "teleported" qubit. Regardless of the specific cause of degradations, the effect of the quantum imperfections imposed on a quantum system is that pure quantum states evolve into mixed quantum states [77]. However, despite its pivotal importance, the accurate modelling of quantum-domain imperfections capable of capturing the effects of the different imperfections on the quantum teleportation process remains an open problem at the time of writing this treatise.

With this in mind, to gain further insights into the behavior of the composite quantum imperfection:

- in Secs. V-A V-D we will provide a rudimentary communications engineering perspective on the theoretical framework of characterizing the effects of quantum decoherence on an arbitrary qubit;

- in Sec. VI, we then complement this theoretical analysis by an experimental perspective, in which we characterize the cumulative quantum imperfections affecting the quantum teleportation relying on an operational quantum chip using the IBM Q platform [78].

The rationale for splitting the analysis into these two steps is that the errors arising in the quantum teleportation owing to imperfect operations strongly depend on the particulars of the technology adopted for representing a qubit. As a consequence, to gain tangible insights into the behavior of the composite quantum imperfection, the adoption of a specific quantum chip technology is inevitable.

Furthermore, with the analysis about to be developed in Secs. V-A V-D in mind we will be able to point out two distinctive features of the quantum imperfections:

i) the quantum imperfections are multiplicative rather than being additive, hence this phenomenon might be deemed more reminiscent of the classical fading effects, rather than of the classical additive noise imposed by the Brownian motion of electrons;

ii) the quantum imperfections exhibit an asymmetric behavior, since they alter the coordinates of the Bloch vector

\footnotetext{
${ }^{22}$ Although the figure represents also the classical noise affecting the classical channel block of Fig. 5 for the sake of completeness, in the following we will focus our attention on the quantum imperfections only.
} 
representing the qubit differently; this phenomenon might be interpreted as a sort of spatial selectivity in terms of Bloch vector coordinates.

These distinctive features of the quantum imperfections will be confirmed by the experimental findings of Sec. VI.

\section{A. Modeling Quantum Decoherence}

When an open quantum system $\mathcal{S}$ of interest - a qubit in our case - interacts with the environment $\mathcal{E}$, they together form the closed quantum system $\mathcal{S E}$ [17], [18], [23], [79]. This closed system $\mathcal{S E}$ evolves according to a unitary transformation $U_{\mathcal{S E}}$ formulated as:

$$
\rho_{\mathcal{S E}}(t)=U_{\mathcal{S E}} \rho_{\mathcal{S E}}(0) U_{\mathcal{S E}}^{\dagger}
$$

with $\rho_{\mathcal{S E}}(\cdot)$ representing the density matrix of the closed quantum system $\mathcal{S E}$, as defined in Sec. II

The status of the system $\mathcal{S}$ of interest can be recovered by tracing out the environment via the partial trace operator $\operatorname{Tr}_{\mathcal{E}}(\cdot)$ over the environment $\mathcal{E}$, which is expressed as:

$$
\rho_{\mathcal{S}}(t)=\operatorname{Tr}_{\mathcal{E}}\left[\rho_{\mathcal{S E}}(t)\right]=\operatorname{Tr}_{\mathcal{E}}\left[U_{\mathcal{S E}} \rho_{\mathcal{S E}}(0) U_{\mathcal{S E}}^{\dagger}\right]
$$

where $\rho_{\mathcal{S}}(t)$ is referred to as the reduced density matrix.

Due to the complex interactions between the system and the environment, in general $\rho_{\mathcal{S}}(t)$ may not be directly related to the initial state $\rho_{\mathcal{S}}(0)$ through a unitary transformation. Furthermore, it is quite a challenge to evaluate (27), since it requires us to determine the dynamics $\rho_{\mathcal{S E}}(t)$ of the composite system $\mathcal{S E}$. Indeed, the status of the environment is always unknown and cannot be controlled in reality.

However, by applying some approximations, it is often possible to derive the approximate evolution of $\rho_{\mathcal{S}}(t)$ vs. time via the master equation formalism of [37]. Accordingly, the evolution of the system $\mathcal{S}$ vs. time can be expressed in the Lindblad form ${ }^{23}$ as a time-local first-order differential equation system of the following form [37], [80]:

$$
\begin{aligned}
\frac{d}{d t} \rho_{\mathcal{S}}(t) & =\overbrace{-\frac{i}{\hbar}\left[H_{s}, \rho_{\mathcal{S}}(t)\right]+}^{\text {unitary evolution }}+ \\
& +\overbrace{\sum_{k}\left(L_{k} \rho_{\mathcal{S}}(t) L_{k}^{\dagger}-\frac{1}{2}\left\{L_{k} \dagger L_{k}, \rho_{\mathcal{S}}(t)\right\}\right)}^{\text {non-unitary evolution }} .
\end{aligned}
$$

In $(28)$, we note the presence of two components. The unitary evolution component depends both on Planck's constant $\hbar$, whose value must be experimentally determined, and on the Hermitian operator $H_{s}$, referred to as the Hamiltonian of the system $^{24}$. In the following we assume $\hbar=1$, without loss

\footnotetext{
${ }^{23}$ In the following, $[A, B]$ denotes the commutator between two operators and it is defined as $[A, B]=A B-B A$. Similarly, $\{A, B\}$ denotes the anticommutator between two operators and it is defined as $\{A, B\}=A B+B A$.

${ }^{24}$ In general, $H_{s}$ in 28 is not identical to the unperturbed free Hamiltonian of the system that would govern the evolution of the system in the absence of any environmental effects [37]. Indeed, the environmental interference typically perturbs the free Hamiltonian, leading to a re-normalization of the energy levels of the system. However, this effect (also known as Lambshift [37]) does not contribute to the non-unitary evolution imposed by the environmental perturbations - it only affects the unitary part of the reduced dynamics.
}

of generality. In the case of a closed system, the knowledge of the Hamiltonian implies having the knowledge of the entire dynamics of the system. The non-unitary evolution component follows from the non-unitary nature of the trace operation used for obtaining the reduced density matrix, and it is driven by the Lindblad operators, $L_{k}$, representing the coupling of the system to its environment.

The Lindblad operators can be constructed from a basis of the vector space associated with the operators acting on the quantum system considered [37]. For a quantum system associated with the dimension $d, d^{2}$ is the dimensionality of the operators vector-space and the Lindblad operators can be constructed from $d^{2}-1$ elements of such a base 25 For onequbit systems, the three Pauli matrices $\left\{\sigma_{k}\right\}_{k=x, y, z}$ reported in Table I. together with the identity matrix, constitute a basis for the vector space associated with the operators. Hence, the Lindblad operators can be constructed from the Pauli matrices [37]. However, the choice of a specific Lindblad operator set is not unique, since unitary transformations of a given Lindblad set describe the same non-unitary evolution of the system. Furthermore, the choice of the specific Lindblad operator set usually depends on the particular type of quantum imperfection under investigation. In the following, we set the Lindblad operators [17], [37], [80] as:

$$
L_{k}=\sqrt{\gamma_{k}} \sigma_{k}, \quad k \in\{x, y, z\} .
$$

In 29], the coefficients $\left\{\gamma_{k}\right\}$ are referred to as decay rates, for the reasons justified in the following, and they depend on the specific interaction between the system $\mathcal{S}$ and the environment $\mathcal{E}$. The choice made in $(29)$ allows us to model an important category of quantum imperfections, generally termed as the depolarizing phenomenon, which imposes errors - such as bitflips, phase-flips and their combinations [17] - typically arising in quantum computation and quantum communications.

Remark 8. We note that upon setting $L_{ \pm}=\sqrt{\gamma_{ \pm}}\left(\sigma_{x} \pm i \sigma_{y}\right)$, it becomes possible to directly model another important category of quantum imperfection, referred to as thermalization noise: a qubit, if left alone for sufficiently long time, will eventually settle into some classical distribution of the basis states $|0\rangle$ and $|1\rangle$ as a consequence of energy exchange with the environment [5], [17]. The depolarizing phenomenon acts on a much shorter time-scale than the thermalization, hence it is the primary imperfection to be considered when modeling quantum teleportation from a communications engineering perspective. Hence, in the following, we restrict our attention to the former. However, it is worthwhile noting that thermalization plays a crucial role in quantum networks, where the quantum states must be stored in quantum memories for fulfilling the communication needs at hand - exemplified by waiting for reply messages from across the network. Hence, we set aside the thermalization modeling for our future work.

Based on these preliminaries, in the next subsections we will review some of the different imperfections from a communications engineering perspective, induced by the interaction

\footnotetext{
${ }^{25}$ The $d^{2}$-th element is the identity matrix, which can be disregarded since it commutes with any $\rho_{\mathcal{S}}(t)$, providing a null contribution to the non-unitary evolution in 28] [37].
} 
between the quantum system and the environment. To this end and without loss of generality, in the following we assume having a Hamiltonian of $H_{s}=\frac{\Omega}{2} \sigma_{z}$, which indicates that the Hamiltonian is dominated by the unperturbed qubit energy splitting $\Omega$ [37], [81]. This assumption is not restrictive, since, as said, the Hamiltonian governs the unitary evolution of the system. Hence, assuming a different expression of the Hamiltonian changes the un-perturbaited behavior of the system but it does not change the analysis of the effects, described by the Lindblad operators, imposed by the environments on the system.

\section{B. Phase Damping}

One of the quantum depolarizing processes with no direct counterpart in the classical world is phase damping, which models the erosion of quantum information without loss of energy. This is one of the most common perturbations in quantum information processing. The phase damping is described by the Lindblad operator $L_{z}=\sqrt{\gamma_{z}} \sigma_{z}$, where again, $\gamma_{z}$ is the decay-rate. Upon substituting it into (28) and by accounting for $L_{z} \dagger L_{z}=\gamma_{z} I$, the resultant time-domain evolution of the system $\mathcal{S}$ may be formulated as:

$$
\begin{aligned}
\frac{d}{d t} \rho_{\mathcal{S}}(t) & =-i\left[\frac{\Omega}{2} \sigma_{z}, \rho_{\mathcal{S}}(t)\right]+ \\
& +\overbrace{L_{z} \rho_{\mathcal{S}}(t) L_{z}^{\dagger}-\frac{1}{2}\left\{L_{z} \dagger L_{z}, \rho_{\mathcal{S}}(t)\right\}}^{\text {non-unitary evolution induced by the Phase Damping }} .
\end{aligned}
$$

Solving (30] as detailed in [82], we find that the diagonal elements $\rho_{\mathcal{S}}^{j J}(t)$ are time-invariant, i.e. we have $\rho_{\mathcal{S}}^{j j}(t)=$ $\rho_{\mathcal{S}}^{j j}(0) \forall t$ with $j=0,1$, whereas the off-diagonal elements are:

$$
\begin{aligned}
& \rho_{\mathcal{S}}^{01}(t)=\rho_{\mathcal{S}}^{01}(0) e^{-\left(i \Omega+2 \gamma_{z}\right) t} \\
& \rho_{\mathcal{S}}^{10}(t)=\rho_{\mathcal{S}}^{10}(0) e^{-\left(-i \Omega+2 \gamma_{z}\right) t}=\left[\rho_{\mathcal{S}}^{01}(t)\right]^{*} .
\end{aligned}
$$

Observe from (31) that an arbitrary qubit obeys a phaseevolution that depends on: i) the energy difference between the states $|0\rangle$ and $|1\rangle$ via the term $\Omega t$ of $(31)$, which induces a rotation around the $\mathrm{z}$-axis of the Bloch sphere, as shown in Fig. 8 [82]; ii) the damping decay rate via the term $2 \gamma_{z} t$ of (31). Since the phase evolution imposed by $\Omega t$ can be compensated by a Phase shift gate $R_{\phi}$ associated with the opposite linear phase of $\phi=\Omega t$ [82], it becomes possible to streamline the noise effects formulated in 31 in the compact form of:

$$
\rho_{\mathcal{S}}(t)=R_{\Omega t} \rho_{\mathcal{S}}(t) R_{\Omega t}^{\dagger}=\left[\begin{array}{cc}
\rho_{\mathcal{S}}^{00}(0) & \rho_{\mathcal{S}}^{01}(0) e^{-2 \gamma_{z} t} \\
\rho_{\mathcal{S}}^{10}(0) e^{-2 \gamma_{z} t} & \rho_{\mathcal{S}}^{11}(0)
\end{array}\right]
$$

Observe in 32 that the off-diagonal elements, $\rho_{\mathcal{S}}^{i j}(t)$ with $i \neq j$, decay exponentially vs. the time at a decay-rate of $\gamma_{z}$. Hence the original information embedded into the initial quantum state represented by these elements exponentially erodes vs. time owing to the phenomenon of phase damping.

In order to further augment the physical interpretation of the phase-damping phenomenon from a communications engineering perspective, let us visualize its effects on the Bloch sphere of Fig. 2 using the Cartesian coordinates of the Bloch

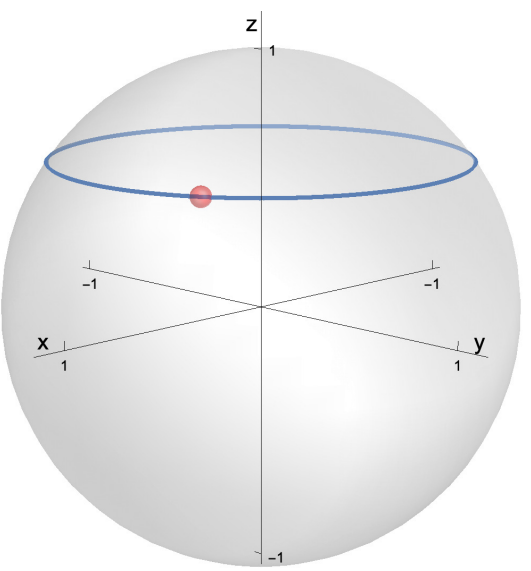

Fig. 8: Bloch sphere representation of the free time-domain evolution of a qubit subject to the Hamiltonian $H_{s}=\Omega \sigma_{z} / 2$, when emerging from the initial pure state of $|\psi(0)\rangle=$ $\sqrt{\frac{1+\sqrt{3}}{2 \sqrt{3}}}|0\rangle+\frac{i+\sqrt{3}}{2 \sqrt{3+\sqrt{3}}}|1\rangle$, located at Bloch vector coordinates $\mathbf{r}(0)=\left[\frac{1}{\sqrt{2}}, \frac{1}{\sqrt{6}}, \frac{1}{\sqrt{3}}\right]$ and indicated by the red point within the figure.

vector $\mathbf{r}=\left[r_{x}, r_{y}, r_{z}\right] \in \mathcal{R}^{3}$ representing the quantum state [18] and introduced in Sec II-H] Specifically, by exploiting (17) and (18), after some algebraic manipulations detailed in [82], the Bloch vector coordinates $\mathbf{r}(t)=\left[r_{x}(t), r_{y}(t), r_{z}(t)\right]$ of the qubit subject to phase damping may be expressed at time instant $t$ as:

$$
\begin{aligned}
& r_{x}(t)=r_{x}(0) e^{-2 \gamma_{z} t} \\
& r_{y}(t)=r_{y}(0) e^{-2 \gamma_{z} t} \\
& r_{z}(t)=r_{z}(0)
\end{aligned}
$$

where $\mathbf{r}(0)=\left[r_{x}(0), r_{y}(0), r_{z}(0)\right]$ represents the Bloch vector at time instant 0 .

Remark 9. Observe in 33 that the phase-damping effects are multiplicative imperfections imposed on the Bloch vector coordinates $r_{x}$ and $r_{y}$ of the quantum state. More explicitily, the phase damping exhibits an asymmetric behavior, since it affects the coordinates of the Bloch vector differently. Specifically, it damps both the $\mathrm{x}$ - and the $\mathrm{y}$-coordinate, i.e. $r_{x}$ and $r_{y}$, while it leaves the $\mathrm{z}$-coordinate, i.e. $r_{z}$, unaltered. This phenomenon might also be interpreted as a sort of spatial selectivity in terms of the Bloch vector coordinates.

Remark 10. We further note that although the phase damping is modelled by the Pauli-Z gate of Table I via the Lindblad operator $L_{z}=\sqrt{\gamma_{z}} \sigma_{z}$, this should not be confused with the pure unitary evolution imposed by the Pauli-Z gate in equation (19), because additionally we have to take into account the non-unitary evolution induced by $L_{z}$ in 30 . More explicitly, similarly to the unitary evolution imposed by the Pauli-Z gate in (19), the phase damping leaves the z-coordinate unaffected, but in contrast to the phase-flipping imposed by the Pauli$\mathrm{Z}$ gate based unitary evolution, the non-unitary evolution represented by $L_{z}$ resulted in a damping of the x- and ycoordinate rather than flipping them. 

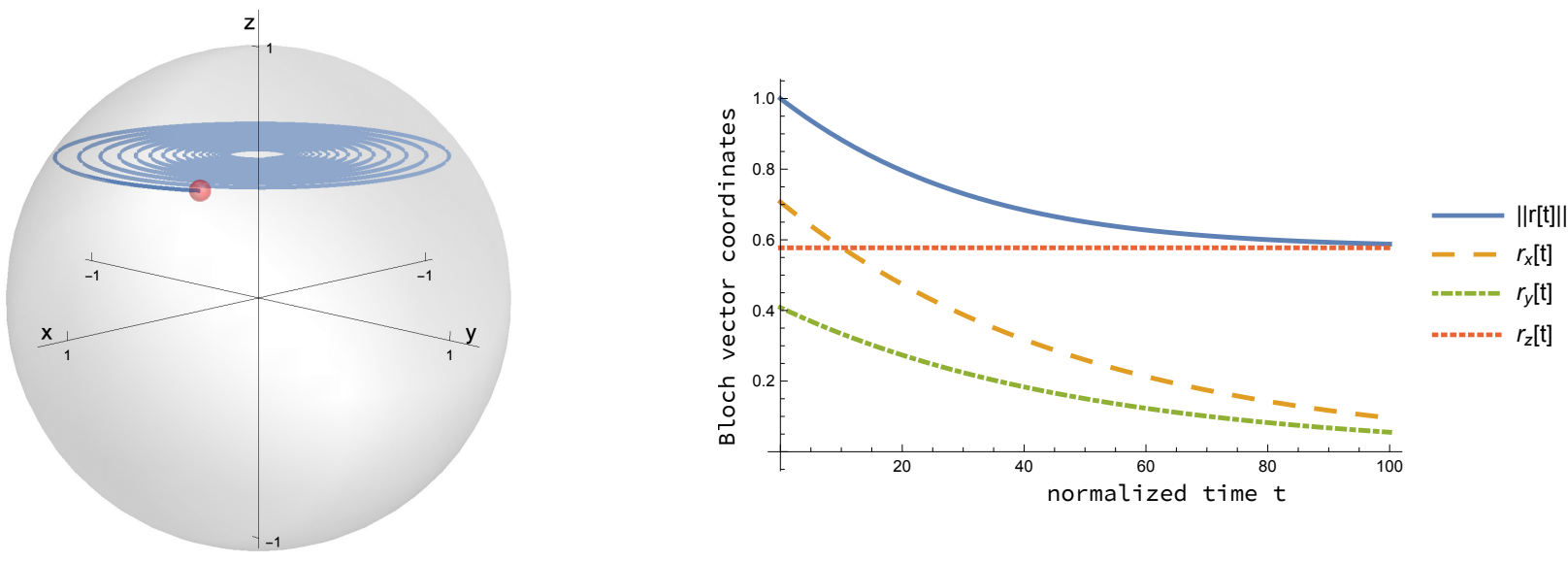

Fig. 9: Phase Damping: time-domain evolution of a qubit subject to the Hamiltonian $H_{s}=\Omega \sigma_{z} / 2$, when emerging from the initial pure state of $|\psi(0)\rangle=\sqrt{\frac{1+\sqrt{3}}{2 \sqrt{3}}}|0\rangle+\frac{i+\sqrt{3}}{2 \sqrt{3+\sqrt{3}}}|1\rangle$ located at the Bloch vector coordinates $\mathbf{r}(0)=\left[\frac{1}{\sqrt{2}}, \frac{1}{\sqrt{6}}, \frac{1}{\sqrt{3}}\right]$ and ending up inside the sphere with unchanged z-coordinate. Left plot: representation of the qubit's time evolution with respect to the Bloch sphere. Right plot: Bloch vector coordinates $\mathbf{r}(t)$ as a function of time, with the phase evolution engendered by the energy difference $\Omega$ in $H_{s}$ appropriately compensated, as detailed in the text.

Remark 11. Observe in (33) that the initial pure state $|\psi(0)\rangle$ is transformed into a mixed state associated with $\|\mathbf{r}(t)\|<1$, as shown in the left plot of Fig. 9 and discussed in the following. Hence, the qubit at time $t>0$ is found within the interior of the Bloch sphere.

To provide a graphical representation of the previous remarks, in Fig. 9, which was originally reported in [82], we portray the time-domain evolution of a qubit emerging from the same initial state as in Fig. 8 and experiencing phase damping.

Specifically, in the left plot of Fig. 9, we portray the qubit's time-domain evolution commencing from the surface of the Bloch sphere and exhibiting phase damping in agreement with (33), where the vertical coordinate $r_{z}$ remains unchanged. By contrast, both the horizontal coordinates $r_{x}$ and $r_{y}$ exponentially decay toward zero. Consequently, the qubit asymptotically evolves towards the mixed state represented by the point $\mathbf{r}=\left[0,0, r_{z}(0)\right]$. Similarly to Fig. 8 , the characteristic orbital evolution in terms of the horizontal coordinates around the vertical axis is a consequence of the linear phase accumulation induced by the system Hamiltonian $H_{s}$ of (31). The phasedamping behavior is further characterized by the right plot of Fig. 9, where the Bloch vector coordinates $\mathbf{r}(t)$ as function of the normalized time are portrayed, but after compensating as in (32) the linear phase-rotation around the vertical axis as induced by $H_{s}$ for explicitly highlighting the spatial selectivity of the phase-damping.

Hence the phase-damping effects can be modeled from a communications engineering perspective using the model of Fig. 10 Specifically, the $\mathrm{x}$ - and the $\mathrm{y}$-coordinate of the qubit are affected by the multiplicative damping, attenuating each coordinate according to an exponential decay governed by $\gamma_{z}$, whereas the z-coordinate of the qubit remains unchanged.

For historical reasons, the phase damping is also often referred to as the $T_{2}$ relaxation process [17], where $T_{2}$ is the

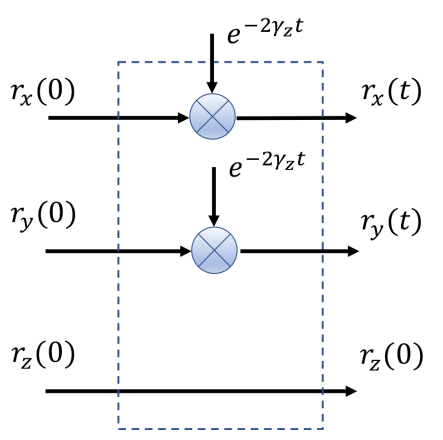

Fig. 10: Phase-Damping Model.

time it takes for a $|+\rangle$ state seen in Fig. 1 to flip to a $|-\rangle$ state with the probability of $e^{-1}[5]$. Since the probability that the qubit is flipped is given by $\frac{1-e^{-\gamma_{z} t}}{2}$ [17], the relationship between the decay rate $\gamma_{z}$ defined in $(29)$ and $T_{2}$ can be expressed as:

$$
T_{2}=-\frac{1}{\gamma_{z}} \ln \left\{\frac{e-2}{e}\right\}
$$

\section{C. $y$-z Damping}

Let us now consider the scenario, when the imperfections are modeled by the Lindblad operator $L_{x}=\sqrt{\gamma_{x}} \sigma_{x}$ [82]. In the following, we refer to this type of depolarizing noise process - also known as bit-flip error process - as $y$ - $z$ damping in analogy with Sec. $\mathrm{V}-\mathrm{B}$ and for the reasons to be further highlighted later in Remark 12.

By solving (28) we may characterize the time-domain evolution of the system $\mathcal{S}$ as in (35), shown at the top of the next page. To elaborate further on (35), we have compensated the phase evolution engendered by $H_{s}$ as in Sec. $\mathrm{V}-\mathrm{B}$ for explicitly highlighting these imperfections. 


$$
\rho_{\mathcal{S}}(t)=\frac{1}{2}\left[\begin{array}{ll}
\rho_{\mathcal{S}}^{00}(0)\left(1+e^{-2 \gamma_{x} t}\right)+\rho_{\mathcal{S}}^{11}(0)\left(1-e^{-2 \gamma_{x} t}\right) & \rho_{\mathcal{S}}^{01}(0)\left(1+e^{-2 \gamma_{x} t}\right)+\rho_{\mathcal{S}}^{10}(0)\left(1-e^{-2 \gamma_{x} t}\right) \\
\rho_{\mathcal{S}}^{10}(0)\left(1+e^{-2 \gamma_{x} t}\right)+\rho_{\mathcal{S}}^{01}(0)\left(1-e^{-2 \gamma_{x} t}\right) & \rho_{\mathcal{S}}^{11}(0)\left(1+e^{-2 \gamma_{x} t}\right)+\rho_{\mathcal{S}}^{00}(0)\left(1-e^{-2 \gamma_{x} t}\right)
\end{array}\right]
$$
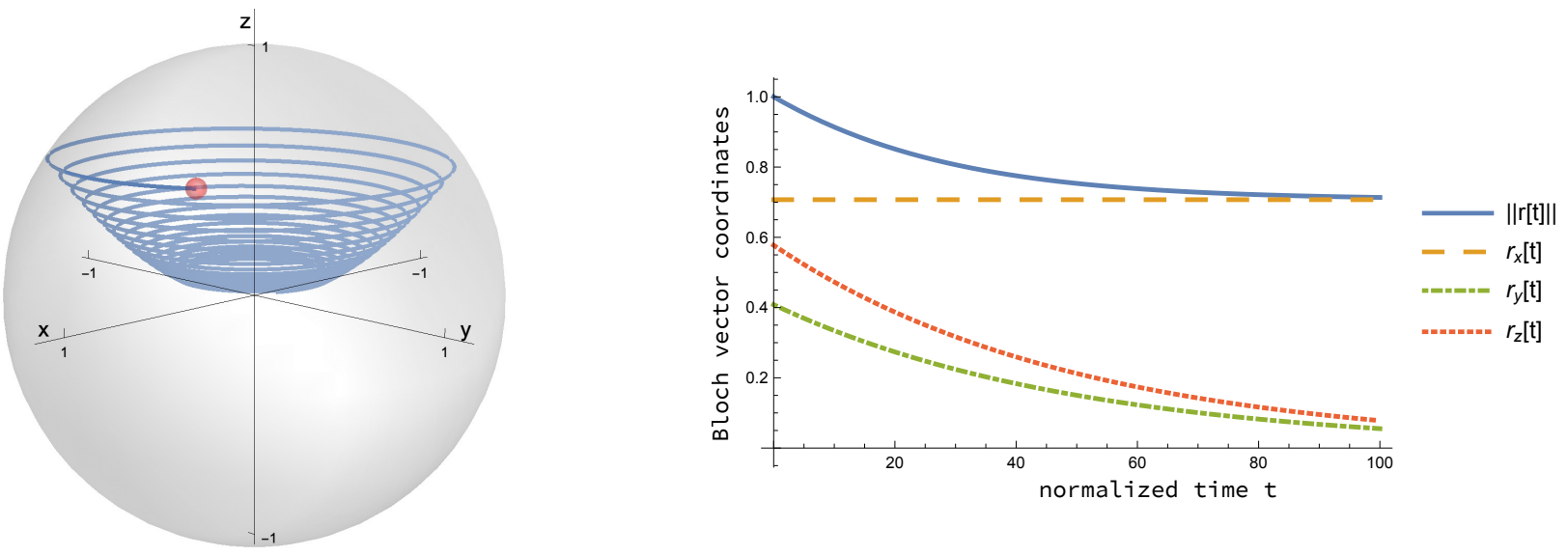

Fig. 11: y-z Damping: time-domain evolution of a qubit with Hamiltonian $H_{s}=\Omega \sigma_{z} / 2$, when emerging from the initial pure state of $|\psi(0)\rangle=\sqrt{\frac{1+\sqrt{3}}{2 \sqrt{3}}}|0\rangle+\frac{i+\sqrt{3}}{2 \sqrt{3+\sqrt{3}}}|1\rangle$ located at the Bloch vector coordinates of $\mathbf{r}(0)=\left[\frac{1}{\sqrt{2}}, \frac{1}{\sqrt{6}}, \frac{1}{\sqrt{3}}\right]$. Left plot: representation of the qubit's time-domain evolution with respect to the Bloch sphere. Right plot: Bloch vector coordinates $\mathbf{r}(t)$ as a function of time, with the phase evolution engendered by the energy difference $\Omega$ in $H_{s}$ appropriately compensated, as detailed in the text.

Similarly to phase damping, by exploiting (17) as well as (18), and accounting for (35), after some further algebraic manipulations, the Bloch vector coordinates $\mathbf{r}(t)=$ $\left[r_{x}(t), r_{y}(t), r_{z}(t)\right]$ of the qubit subject to $\mathrm{y}-\mathrm{z}$ damping evolve in the time-domain according to the following relationship:

$$
\begin{aligned}
& r_{x}(t)=r_{x}(0), \\
& r_{y}(t)=r_{y}(0) e^{-2 \gamma_{x} t}, \\
& r_{z}(t)=r_{z}(0) e^{-2 \gamma_{x} t},
\end{aligned}
$$

where $\mathbf{r}(0)=\left[r_{x}(0), r_{y}(0), r_{z}(0)\right]$ represents the Bloch vector at time instant 0 .

Remark 12. Similarly to phase damping, observe in 36 that the $\mathrm{y}-\mathrm{z}$ damping effects are also multiplicative imperfections imposed on the Bloch vector coordinates of the quantum state. Furthermore and similarly to the phase damping, the $y-z$ damping exhibits an asymmetric behavior, since it affects the individual Bloch vector coordinates differently. Specifically, it damps exponentially both the $\mathrm{y}$ - and the $\mathrm{z}$-coordinate, i.e. $r_{y}$ and $r_{z}$, while it leaves the X-coordinate, i.e. $r_{x}$, unaffected. This phenomenon might be interpreted again as a sort of spatial selectivity in terms of the Bloch vector coordinates. Moreover, similarly to phase damping, the initial pure state $|\psi(0)\rangle$ is transformed into a mixed state associated with $\|\mathbf{r}(t)\|<1$ for $t>0$.

Remark 13. We note furthermore that although the $y-z$ damping is modelled by the Pauli-X gate of Table I via the Lindblad operator $L_{x}=\sqrt{\gamma_{x}} \sigma_{x}$, we should not confuse the unitary evolution imposed by the Pauli-X gate and highlighted in equation (19) with the non-unitary evolution induced by $L_{x}$ in (28). More explicitly, similarly to the unitary evolution

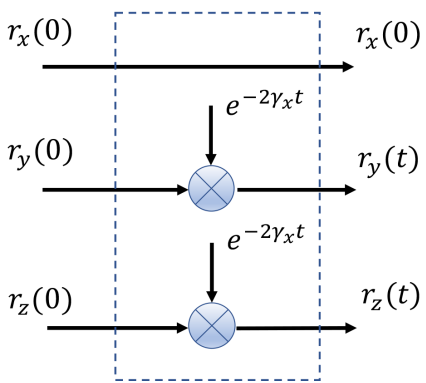

Fig. 12: y-z Damping Model.

imposed by the Pauli-X gate in (19), the $y-z$ damping leaves the $\mathrm{X}$-coordinate unaffected, but in contrast to the bit-flipping imposed by the Pauli-X gate based unitary evolution, the nonunitary evolution represented by $L_{x}$ resulted in an exponential damping of the $\mathrm{y}$ - and the $\mathrm{z}$-coordinate rather than flipping them.

To visualize the above discussion, in Fig. 11 we portray the time-evolution of a qubit emerging from the same initial state as in Figs. 8 as well as 9 and subject to $y-z$ damping.

Specifically, in the left plot, we portray the qubit's timedomain evolution commencing from the surface of the Bloch sphere. But then as time elapses, we observe that all the coordinates decay to zero, which seems to contradict (36). However, this contradiction is only illusory, since in the left plot, we do not compensate the orbital evolution of the $\mathrm{x}$ and y-coordinate around the z-axis induced by $\Omega$ in $H_{s}$. This is confirmed by the right plot of Fig. 11, where we have appropriately compensated this phase evolution as in (32) 

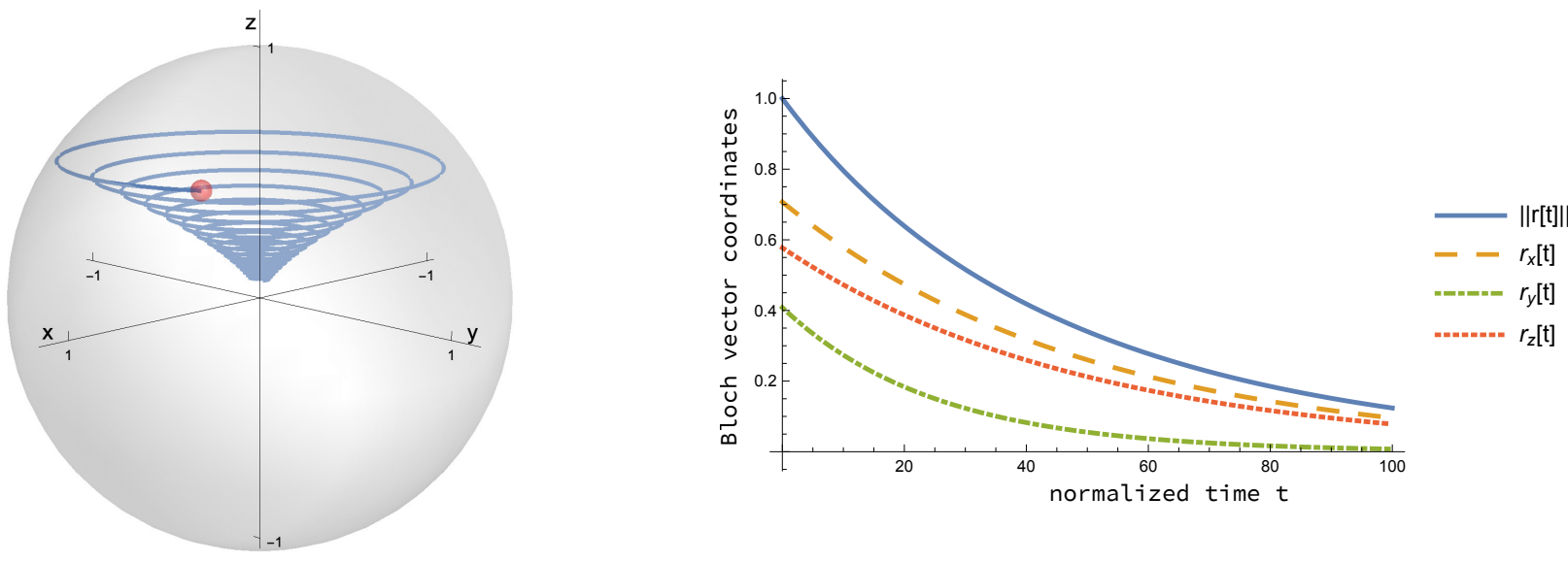

Fig. 13: Combined y-z-phase damping: time-domain evolution of a qubit subject to the Hamiltonian $H_{s}=\Omega \sigma_{z} / 2$. when emerging from initial pure state of $|\psi(0)\rangle=\sqrt{\frac{1+\sqrt{3}}{2 \sqrt{3}}}|0\rangle+\frac{i+\sqrt{3}}{2 \sqrt{3+\sqrt{3}}}|1\rangle$ located at the Bloch vector coordinates $\mathbf{r}(0)=$ $\left[\frac{1}{\sqrt{2}}, \frac{1}{\sqrt{6}}, \frac{1}{\sqrt{3}}\right]$. Left plot: representation of the qubit's time evolution with respect to the Bloch sphere. Right plot: Bloch vector coordinates $\mathbf{r}(t)$ as a function of time, with the phase evolution engendered by the energy difference $\Omega$ in $H_{s}$ appropriately compensated, as detailed in the text.

for explicitly highlighting the spatial selectivity of the $y-z$ damping. In agreement with (36), the $x$-coordinate remains unaltered, whereas the $y$ - and the z-coordinates exponentially decay towards zero with a decay-rate of $\gamma_{x}$. Hence, the qubit asymptotically evolves towards the mixed state represented by the point $\mathbf{r}=\left[r_{x}(0), 0,0\right]$.

Based on the above discussions, the $y-z$ damping effects can be modeled from a communications engineering perspective using the model of Fig. 12. Specifically, the y- and the zcoordinate of the qubit are affected by a multiplicative damping, attenuating each coordinate according to an exponential decay governed by $\gamma_{x}$, whereas the x-coordinate of the qubit remains unchanged.

\section{Combined y-z-Phase Damping}

Based on the discussions of Secs. $\mathrm{V}-\mathrm{B}$ and $\mathrm{V}-\mathrm{C}$, the spontaneous question arises: "is the multiplicative nature of the quantum imperfections modeled in (28) general?"

To gain further insights concerning this question, we analyze the behavior of the composite imperfections, namely when the imperfections are modeled by a combination of the two Lindblad operators $L_{x}=\sqrt{\gamma_{x}} \sigma_{x}$ and $L_{z}=\sqrt{\gamma_{z}} \sigma_{z}$. In the following, we refer to this type of depolarizing process as combined $y$-z-phase damping in analogy with Secs. V-B V-C

As before, by compensating for the phase evolution induced by the Hamiltonian $H_{s}=\frac{\Omega}{2} \sigma_{z}$, commencing from [28), after some algebraic manipulations we arrive at:

$$
\begin{aligned}
& r_{x}(t)=r_{x}(0) e^{-2 \gamma_{z} t}, \\
& r_{y}(t)=r_{y}(0) e^{-2\left(\gamma_{x}+\gamma_{z}\right) t}, \\
& r_{z}(t)=r_{z}(0) e^{-2 \gamma_{x} t} .
\end{aligned}
$$

Remark 14. Similarly to the previous scenarios, observe in (37) that the combined y-z-phase damping effects impose multiplicative imperfections on the Bloch vector coordinates of the quantum state. Furthermore, the combined y-z-phase damping exhibits an asymmetric behavior, since it affects the individual coordinates of the Bloch vector differently. Specifically, it damps all the three coordinates exponentially, but at different decay rates. This phenomenon might again be interpreted as a spatial selectivity in terms of Bloch vector coordinates, with the initial pure state $|\psi(0)\rangle$ being transformed into a mixed state.

Remark 15. Similarly to the previous subsections, we should avoid confusing the unitary evolution imposed by a sequence of the Pauli-X and Pauli-Z gates with the non-unitary evolution induced by a combination of the two Lindblad operators $L_{x}$ and $L_{z}$ present in (28).

The multiplicative nature of the combined imperfections is further confirmed by Fig. 13, showing the time evolution of a qubit subject to combined y-z-phase damping. Specifically, as done in the previous subsections, we consider a qubit emerging from the initial pure state of $|\psi(0)\rangle$ whose Bloch vector coordinates are $\mathbf{r}(0)=\left[\frac{1}{\sqrt{2}}, \frac{1}{\sqrt{6}}, \frac{1}{\sqrt{3}}\right]$. The left plot shows the representation of the qubit's time-domain evolution starting from the surface of the Bloch sphere. Similarly to the left plot of Fig. 11, all the Bloch vector coordinates evolve towards zero. However, by comparing the two figures, we recognize that in the y-z-phase damping scenario, the evolution of the quantum state towards the center of the sphere is faster. This is in agreement with (37). In fact, as shown by the right plot in Fig. 13 , by appropriately compensating the phase evolution induced by $H_{s}$, all the Bloch coordinates exponentially decay toward zero, with a decay-rate of: i) either $\gamma_{z}$ or $\gamma_{x}$ for the $\mathrm{x}$ - and z-coordinate, respectively; ii) the sum of $\gamma_{x}$ and $\gamma_{z}$ for the y-coordinate.

Based on the above discussions, the combined y-z-phase damping effects can be modeled from a communications en- 


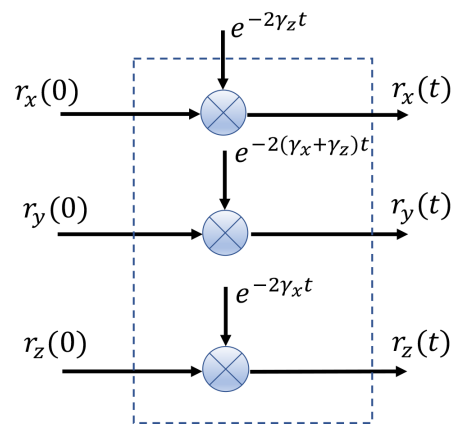

Fig. 14: Combined y-z-Phase Damping Model.

gineering perspective using the model of Fig. 14 Specifically, all the qubit coordinates are affected by the multiplicative damping, with an exponential decay rate that differs among the different coordinates.

Based on the reasoning above, it may be readily shown that a quantum imperfection modeled by the combination of all the three Lindblad operators $-L_{x}=\sqrt{\gamma_{x}} \sigma_{x}, L_{y}=\sqrt{\gamma_{y}} \sigma_{y}$ and $L_{z}=\sqrt{\gamma_{z}} \sigma_{z}$ - changes the Bloch vector coordinates of a qubit according to the following relationship:

$$
\begin{aligned}
& r_{x}(t)=r_{x}(0) e^{-2\left(\gamma_{y}+\gamma_{z}\right) t}, \\
& r_{y}(t)=r_{y}(0) e^{-2\left(\gamma_{x}+\gamma_{z}\right) t}, \\
& r_{z}(t)=r_{z}(0) e^{-2\left(\gamma_{x}+\gamma_{y}\right) t} .
\end{aligned}
$$

Consequently, all the previous considerations continue to hold.

Remark 16. The above analysis revels that the decoherence affects the three spatial directions by a similar mechanism, namely by multiplying the Bloch coordinates of the qubit with an exponential function. However, the exponent is different for the three coordinates, dictated by the complex interaction between the system and the environment. From a communications engineering perspective, the decoherence may be represented as in Fig. 15 .

\section{IBM Q EXPERIMENTAL RESULTS}

In this section we report the results of teleportation experiments relying on a real quantum computer through the IBM Q platform [29]. Our objective is to gain experimental insights concerning the composite quantum imperfections affecting the teleported qubit.

Specifically, we perform quantum process tomography [83][89] for fully characterizing the dynamics of teleportation. At the time of writing the IBM Q project does not allow us to account for the channel effects within the entanglement distribution, resulting in a setup similar to the one used in [25] where the entangled-photon source and the Bell-state measurement are performed at the same location on the ground. Nevertheless, the experiments allow us to evaluate the deleterious effects of cumulative imperfections encountered during entanglement generation, as well as by imperfect quantum gates at both the source and destination, and finally the decoherence effects.

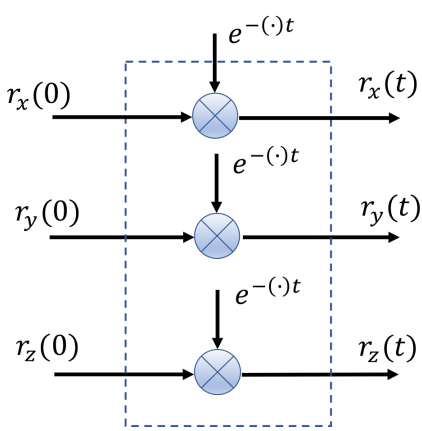

Fig. 15: Arbitrary Decoherence Process Model.

For the quantum circuit depicted in Figure 3, we perform over 8 million quantum process tomography ${ }^{26}$ experiments using the 5-qubits IBM Tenerife ibmqx4 quantum processor during the period of January 14th to January 19th 2019. The quantum state to be teleported was placed in the chip-qubit $q[0]$ and the EPR pair was created ${ }^{27}$ between chip-qubits $q[1]$ and $q[2]$.

\section{A. Teleporting a basis state}

In Figure 16, we evaluate the effects of the cumulative quantum imperfections when the qubit to be teleported is the pure state $|\psi\rangle=|0\rangle$. In the absence of imperfections, the teleported qubit would coincide with the original qubit $|0\rangle$, and it will be placed at the Bloch vector coordinates of $[0,0,1]$ (green dot in Fig. 16d). However, as discussed in Sec. V, real quantum systems constitute open physical systems - where decoherence arises due to the interactions with the environment. Hence, the teleported qubit differs from the original qubit $|0\rangle$ and, in agreement with the theoretical results of Sec. V the teleported qubit is no longer in a pure state, but it is rather in a mixed state laying inside the Bloch sphere.

Specifically, we characterize the effects of the cumulative imperfections using the marginal Cumulative Distribution Functions (CDFs) of the Bloch vector coordinates of the teleported state, as seen in Figs. 16a 16c.

Regarding the Bloch x-coordinate, Fig. 16a shows its marginal CDF $F_{R_{x}}\left(r_{x}\right)$, where, by definition, $F_{R_{x}}\left(r_{x}\right) \triangleq$ $P\left(R_{x} \leq r_{x}\right)$, i.e., $F_{R_{x}}\left(r_{x}\right)$ is the probability of the $\mathrm{x}$ coordinate of the teleported state being smaller than or equal to $r_{x}$. We observe that, with probability roughly equal to one, the $\mathrm{x}$-coordinate assumes values within the interval $(-0.044,0.056)$, with an average value $\mu_{x}$ around 0 . Furthermore, the figure also shows the distribution that better fits with the experimental data, obtained with the aid of the Mathematica FindDistribution and EstimatedDistribution packages. Observe that the $\mathrm{x}$-coordinate of the teleported qubit can be roughly modeled by the normal distribution $\mathcal{N}\left(\mu_{x}, \sigma_{x}\right)$

\footnotetext{
${ }^{26}$ Quantum process tomography is sensitive to the so called state preparation and measurement (SPAM) errors. Hence, the experiment results discussed in the following are inevitably affected also by SPAM errors.

${ }^{27} \mathrm{By}$ applying a $\mathrm{H}$ gate on $q[0]$, followed by a CNOT gate on $q[1]$ with $q[0]$ as control.
} 


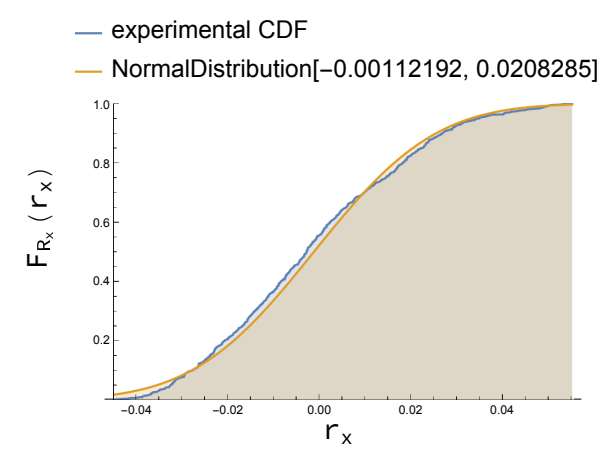

(a) Marginal CDF $F_{R_{x}}\left(r_{x}\right)$ of the Bloch $\mathrm{x}$ coordinate.

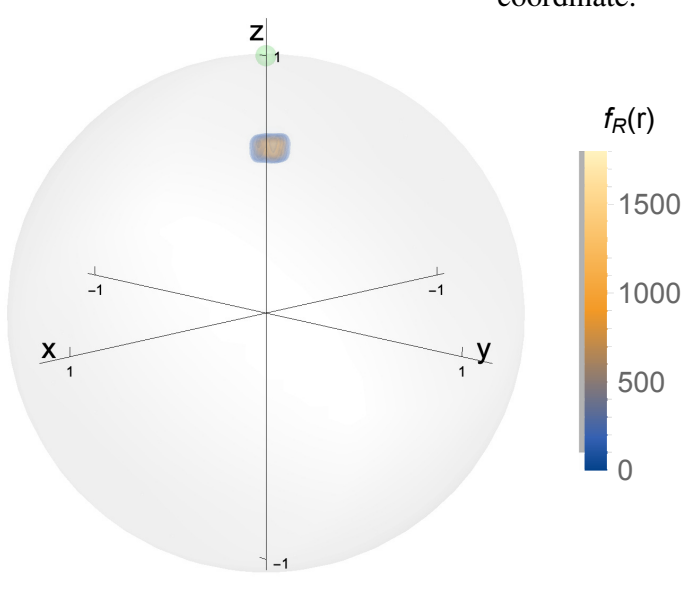

(d) Density plot of the experimental joint PDF $f_{\mathbf{R}}(\mathbf{r})$ of the Bloch vector $\mathbf{r}=\left[r_{x}, r_{y}, r_{z}\right]$.

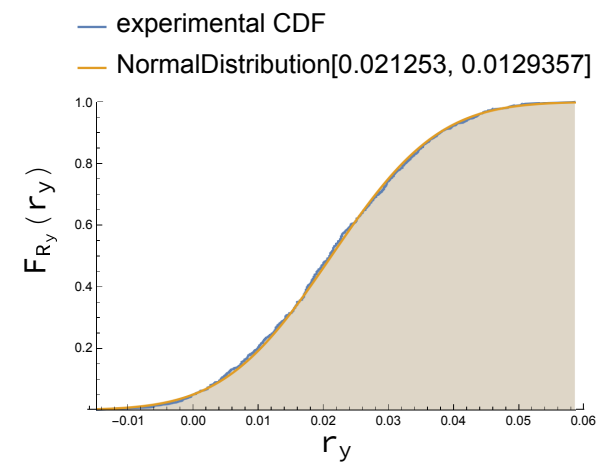

(b) Marginal CDF $F_{R_{y}}\left(r_{y}\right)$ of the Bloch ycoordinate.

Fig. 16: Teleportation with 5-qubits IBM Tenerife ibmqx4 quantum processor between chip-qubits $q[0]$ and $q[2]$. Initial pure state $|\psi\rangle=|0\rangle$ located at the Bloch vector coordinates $\mathbf{r}=[0,0,1]$ (green dot in Fig. 16d).

associated with $\mu_{x} \simeq 0$ and $\sigma_{x} \simeq 0.021$. However, we note that rigorous distribution fitting theory would result in a low confidence metric, because there are intervals, where there is a consistent shape-deviation between the theory and measurement.

Regarding the Bloch y-coordinate, whose CDF is shown in Fig. $16 \mathrm{~b}$ the results show that it can also be roughly modeled by a normal distribution with a variance of $\sigma_{y} \simeq 0.013$. However, in this case, there is a slight drifting toward positive values, with an average value $\mu_{y}$ of around 0.02 .

Finally, regarding the Bloch z-coordinate whose CDF is shown in Fig. 16c we observe that the $\mathrm{z}$-coordinate of the teleported qubit shrinks from the original value of 1 to an average value $\mu_{z} \simeq 0.66$. Furthermore, with a probability of approximately zero, the z-coordinate assumes values outside the interval $(0.605,0.712)$ and it can be modeled by a normal distribution having a variance of $\sigma_{z} \simeq 0.022$.

The drift of the qubit from the original pure state $|0\rangle$ to a mixed state laying in the interior of the Bloch sphere becomes evident in Fig. 16d, where we report the density plot of the experimental joint Probability Density Function (PDF) of the Bloch vector coordinates of the teleported qubit. Specifically, the experimental data indicate that the teleported qubit lays within a sphere roughly centered at coordinates of $\left[\mu_{x}=0, \mu_{y}=0.02, \mu_{z}=0.66\right]$ with radius around 0.1 . The probability of obtaining a value within such a sphere is clearly given by the joint PDF depicted in Fig. 16d.

We note furthermore that the effect of the quantum imperfections on the different coordinates is not independent. Indeed, as shown in Fig. 16e, there exists a correlation between the effects inflicted upon the $\mathrm{x}$ - and $\mathrm{y}$-coordinate. Specifically, a drift toward positive values of the $\mathrm{x}$-coordinate is coupled with a drift toward lower values of the y-coordinate.

Remark 17. This first set of experiments seems to suggest, in agreement with the theoretical analysis developed in Sec. V. that the cumulative imperfections are multiplicative, resulting in a pure state being transformed into a mixed state. However, to gain more general insights into the composite quantum imperfections, in the next sub-section we carry out a quantum process tomography experiment for characterizing the dynamics of the teleportation of superposed quantum states.

\section{B. Teleporting superposed states}

In Figure 17, we evaluate the effects of the cumulative quantum imperfections when the qubit to be teleported is in the pure state of $|\psi\rangle=|+\rangle=(|0\rangle+|1\rangle) / \sqrt{2}$. Ideally, the 


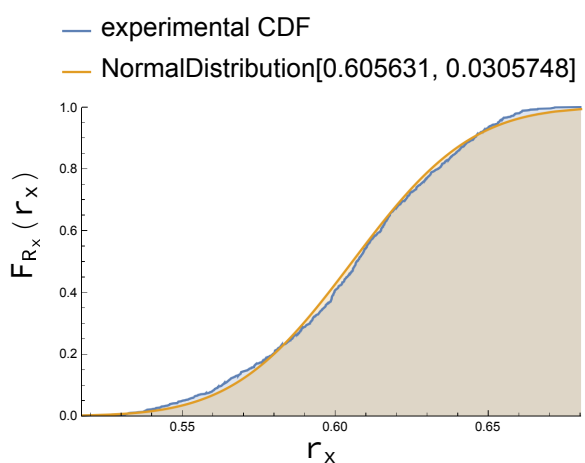

(a) Marginal CDF $F_{R_{x}}\left(r_{x}\right)$ of the Bloch $\mathrm{x}$ coordinate.

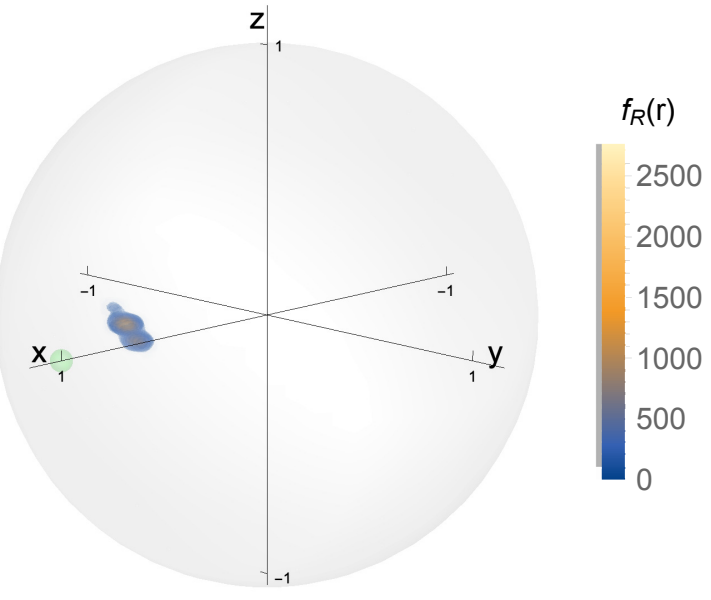

(d) Density plot of the experimental joint PDF $f_{\mathbf{R}}(\mathbf{r})$ of the Bloch vector $\mathbf{r}=\left[r_{x}, r_{y}, r_{z}\right]$.

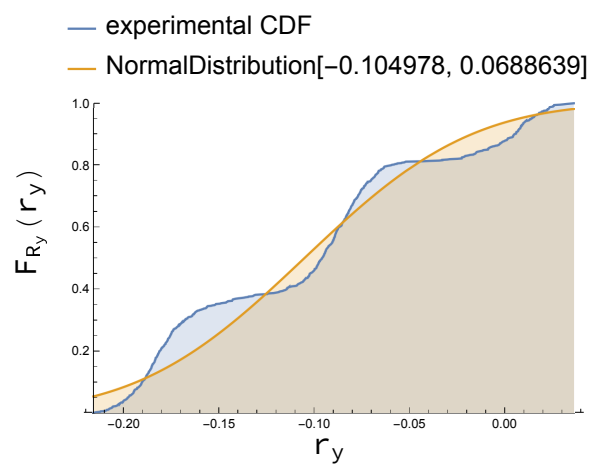

(b) Marginal CDF $F_{R_{y}}\left(r_{y}\right)$ of the Bloch ycoordinate.

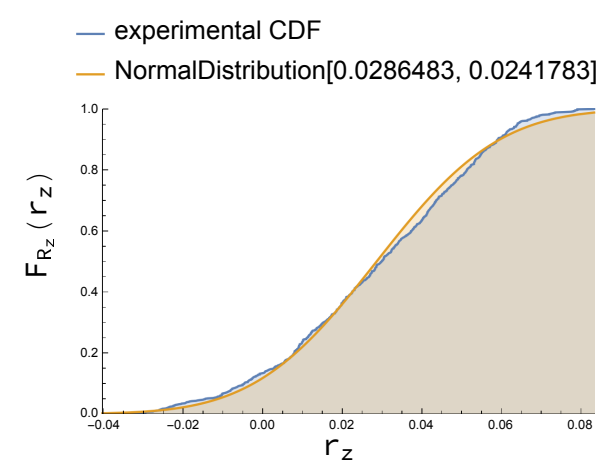

(c) Marginal CDF $F_{R_{z}}\left(r_{z}\right)$ of the Bloch zcoordinate.

Fig. 17: Teleportation with 5-qubits IBM Tenerife ibmqx4 quantum processor between chip-qubits $q[0]$ and $q[2]$. Initial pure state $|\psi\rangle=|+\rangle$ with Bloch vector coordinates $\mathbf{r}=[1,0,0]$ (green dot in Fig. 17d).

teleported qubit would coincide with the original qubit $|+\rangle$ placed at the Bloch vector coordinates of $[1,0,0]$ (green dot in Fig. 17d). However, due to the imperfections and in agreement with the theoretical results of Sec. V, the teleported qubit is corrupted into a mixed state laying inside the Bloch sphere.

Let us now focus our attention on Figs. 17a $17 \mathrm{c}$, where we characterize the cumulative imperfections through the marginal CDFs of the Bloch vector coordinates of the teleported qubit and the results of Sec. VI-A are confirmed. Specifically, Fig. 17a also provides clear evidence of the multiplicative nature of the cumulative imperfections: the $\mathrm{x}$ coordinate of the teleported qubit shrinks from the original value of 1 to an average value of about $\mu_{x}=0.61$. Furthermore, the probability that the $\mathrm{x}$-coordinate of the teleported qubit assumes values outside the interval $(0.517,0.681)$ is approximately zero. To elaborate a little further, similarly to Sec. VI-A the experimental distributions of the $\mathrm{x}$-coordinate and the z-coordinate seen in Fig. $17 \mathrm{c}$ are reminiscent of normal distributions, with parameters $\left(\mu_{x}=0.61, \sigma_{x}=0.03\right)$ and ( $\left.\mu_{z}=0.03, \sigma_{x}=0.02\right)$, respectively.

Regarding the $\mathrm{y}$-coordinate, whose $\mathrm{CDF}$ is shown in Fig. 17b we observe a slight drifting toward negative values and the distribution exhibits three local peaks roughly at
$-0.18,-0.09$, and 0.01 . We believe the peaks are induced by the quantum device calibration procedures [90]. Indeed, the experimental campaign lasted six days, and roughly once a day the IBM chip went off-line for full calibration. If we limit the analysis to the data collected between two consecutive calibrations, we might surmise that each coordinate, including the y-coordinate, can be roughly modeled by a normal distribution. Furthermore, it is worthwhile observing that, according to the Mathematica FindDistribution and EstimatedDistribution packages, the normal distribution has the best fit with the experimental data, given the limited set of hypothesis-distributions, even though the rigorous goodnessof-fit metrics are low. Further research is needed to fully understand the effects of the calibration procedures on the cumulative imperfections.

The peaks along the $y$-coordinate can be easily spotted in Fig. 17d where we portray the density plot of the experimental joint PDF of the Bloch vector coordinates of the teleported state. Furthermore, similarly to Sec. VI-A, the effects of the cumulative imperfections on the different coordinates are not independent. For instance, as shown in Fig. 17e there exists a correlation between the $y$ - and $\mathrm{z}$-coordinate, where the positive values of the $\mathrm{z}$-coordinate are more likely to be associated with 


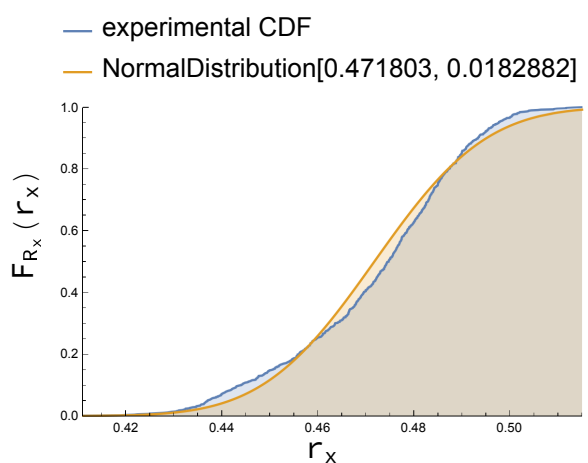

(a) Marginal CDF $F_{R_{x}}\left(r_{x}\right)$ of the Bloch $\mathrm{x}$ coordinate.

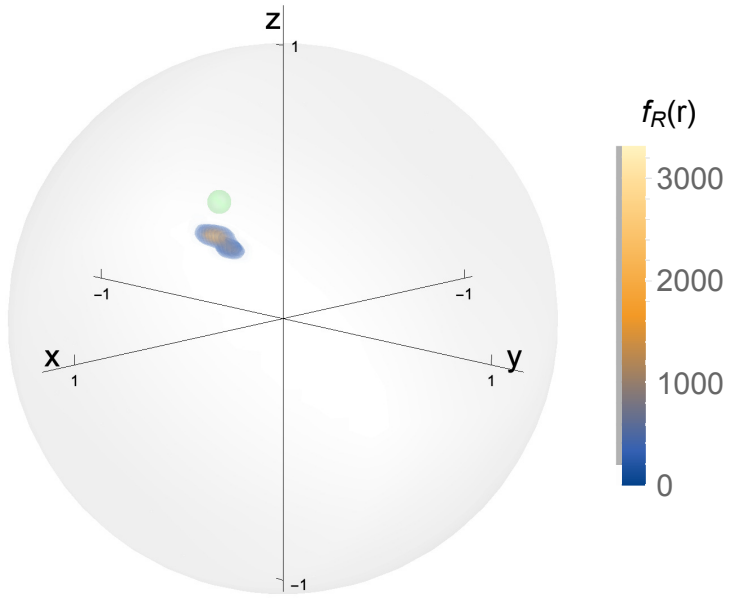

(d) Density plot of the experimental joint PDF $f_{\mathbf{R}}(\mathbf{r})$ of the Bloch vector $\mathbf{r}=\left[r_{x}, r_{y}, r_{z}\right]$. (b) Marginat

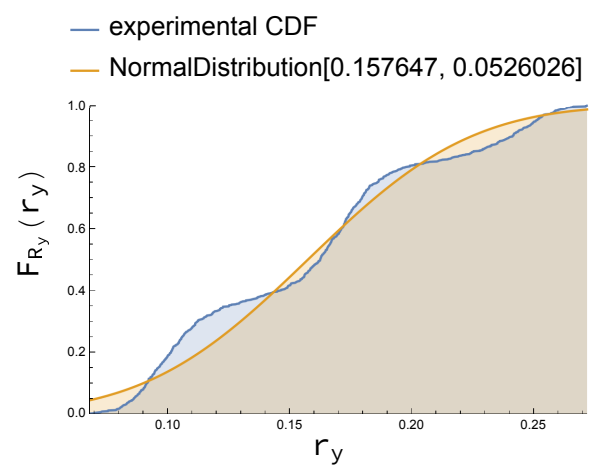

(b) Marginal CDF $F_{R_{y}}\left(r_{y}\right)$ of the Bloch y-

Fig. 18: Teleportation with 5-qubits IBM Tenerife ibmqx4 quantum processor between chip-qubits $q[0]$ and $q[2]$. Initial pure state $|\psi\rangle=\sqrt{\frac{1+\sqrt{3}}{2 \sqrt{3}}}|0\rangle+\frac{i+\sqrt{3}}{2 \sqrt{3+\sqrt{3}}}|1\rangle$ with Bloch vector coordinates $\mathbf{r}=\left[\frac{1}{\sqrt{2}}, \frac{1}{\sqrt{6}}, \frac{1}{\sqrt{3}}\right]$ (green dot in Fig. 18d).

lower values of the $y$-coordinate.

Remark 18. This second set of experiments confirms Remark 17. Specifically, we observe also for a superposed state that the cumulative imperfections are multiplicative, which is in agreement with the theoretical analysis developed in Sec. V Nevertheless, to provide further insights, in the following we consider a superposed quantum state with all the three Bloch vector coordinates being different from zero.

In Figure 18, we evaluate the effects of the cumulative quantum imperfections, when the qubit to be teleported is the pure state $|\psi\rangle$, placed at the Bloch vector coordinates of $\left[r_{x}^{i n}=1 / \sqrt{2}, r_{y}^{i n}=1 / \sqrt{6}, r_{z}^{i n}=1 / \sqrt{3}\right]$ (green dot in Fig. 18d), as in Sec. V. As in the previous experiments, due to the composite imperfections, the teleported qubit is found in a mixed state.

Finally, in Figs. 18a 18c, we characterize the cumulative effects through the marginal CDFs of the Bloch vector coordinates of the teleported qubit. The results of the previous experiments are confirmed: i) reasonable fitting between the experimental distribution and the Gaussian one; ii) multiplicative nature of the quantum imperfections.
However, with this last set of experiment we gain some additional insights concerning the theoretical system model.

Specifically, we observe from Figs. $18 \mathrm{a} 18 \mathrm{c}$ that the $\mathrm{x}-$ coordinate, $\mathrm{y}$-coordinate and $\mathrm{z}$-coordinate of the teleported qubit shrink from the original values of $r_{x}^{i n}, r_{y}^{i n}, r_{z}^{i n}$ to average values of about $\mu_{x}=0.47, \mu_{y}=0.16, \mu_{z}=0.39$.

By analyzing the average attenuations experienced by the $\mathrm{x}$ and the $\mathrm{z}$-coordinate, we note that they are similar, $\mu_{x} / r_{x}^{i n} \simeq$ $\mu_{z} / r_{z}^{i n} \simeq 0.67$, which is also in line with the first two experiments. Explicitly, in Fig. 16c we have $\mu_{z} /\left(r_{z}^{i n}=1\right) \simeq 0.66$ for the z-coordinate and in Fig. $17 \mathrm{a} \mu_{x} /\left(r_{x}^{i n}=1\right) \simeq 0.61$ for the $\mathrm{X}$-coordinate.

As regards to the y-coordinate, we observe that it is subjected to a stronger average attenuation, i.e. $\mu_{y} / r_{y}^{i n} \simeq 0.39$, about twice that of the $\mathrm{x}$ - and $\mathrm{z}$-coordinate.

Remark 19. This third experiment suggests that the cumulative imperfections behave in agreement with the $y$-z-phase damping model of Sec. V-D as summarized in Fig. 14, in conjunction with equal decay rates, i.e. $\gamma_{x}=\gamma_{z}$, as also confirmed by the first two experiments. However, further research is needed to confirm this hypothesis, along with the specific values of the decay rates and their relationship with 
the statistical parameters governing the joint distribution of the teleported Bloch vector coordinates. The correlation of the teleported Bloch vector coordinates also has to be further investigated in order to characterize the decay rates.

\section{OUTLOOK}

Quantum teleportation is the core functionality of the Quantum Internet, which facilitates the "transfer" of qubits without the physical transfer of the particle storing the qubit. Unfortunately, the quantum teleportation process is gravely affected by the quantum imperfections, as analyzed in Sec. V

To this aim, Secs. $\mathrm{V}$ and $\mathrm{VI}$ provided a first step toward the modeling of the quantum imperfections arising during the quantum teleportation process from a communications engineering perspective. Both the theoretical analysis and the experimental campaign allowed us to gain important insights into the behavior of the composite quantum imperfections. Specifically, they revealed that the imperfections are multiplicative and they also exhibit an asymmetric behavior, affecting the Bloch vector coordinates of a qubit differently. Moreover, the composite quantum imperfections obey the theoretical y-z-phase damping model analyzed in Sec. V-D although this has to be further investigated.

At this stage a natural question arises: "how can we generalize the communication system model proposed in Fig. 5 for the quantum teleportation in order to account for all quantum imperfections?"

To answer to this question, we first summarize some of the considerations developed in the previous sections. Specifically, according to the communication system model given in Fig. 5 the noise acts on:

i) the Entanglement Generator \& Distributor super-block ii) the Quantum Pre/Post-Processing blocks.

The imperfection imposed at the Entanglement Generator \& Distributor affects both the generation and the distribution of the entangled pair at Alice and Bob. This in turn implies that the imperfect generation and/or distribution processes provide eventually Alice and Bob with a pair of "imperfect" entangled qubits - rather than a pair of maximally entangled qubits.

Furthermore, even if we assume having a perfect Entanglement Generator \& Distributor super-block, contamination is still present at the Quantum Pre/Post-Processing blocks due to decoherence and/or imperfect quantum operations.

In the following, we discuss the open problems to be circumvented within these communication system blocks.

\section{A. Mitigating imperfections in Quantum Pre/Post-Processing}

Intuitively, to enhance the Quantum Pre/Post-Processing blocks, we can modify the system model of Fig. 5 by introducing a system block at Alice's side that imposes some redundancy into the quantum information sequence. This redundancy can be exploited at Bob's side by a complementary system block to mitigate the imperfections. These two system blocks are the well-known channel-encoder and channeldecoder pair within a classical communication system model [76].
However, a one-to-one mapping between a classical channel-encoder (decoder) and its quantum equivalent cannot be taken for granted. In fact, the no-cloning theorem prevents the adoption of classical error correction techniques relying on the capability of copying the classical information. Hence, specifically designed Quantum Error Correction (QEC) techniques have to be used [71], [91].

QEC techniques are not the only strategies that can be used to mitigate the impact of the environment on a quantum system. Rather than storing data in individual qubits, a decoherence-free subspace (DFS) stores data in multiple qubits, choosing a particular aspect of the system that is less affected by one or more important environmental factors [92]. For example, simply allowing the phase of an individual physical qubit to represent the phase of our data leaves it vulnerable to noise affecting that phase. Instead, we can use the phase difference between two qubits, set up so that the noise that causes phase errors affects both qubits equally, maintaining their phase difference. Mathematically, we say that the DFS stores the information in subspaces of the Hilbert space associated with the quantum system that are least affected by the interaction between the system and its environment [93], [94]. However, DFS techniques are usually affected by the difficulty of identifying the decoherence-free subspaces for more complex systems [94]. Another class of techniques mitigating the environmental effects is constituted by the dynamical coupling (DD) [95], [96]. The idea is to manipulate the non-unitary component of open-system evolutions through the application of external controllable interactions, e.g. a sequence of radio-frequency pulses [96]. However, DD requires near-perfect suppression of the experimental imperfections by the robust design of the sequence [93].

The aforementioned techniques have different pros and cons, by adding different types and different amounts of overheads to any quantum device that uses them. The overhead may consist of additional qubits (particularly in QEC) or additional controls or gate operations (particularly in DD, but also in QEC) [93]. Hence, further research is needed. Indeed, it constitutes an open problem to determine whether a single quantum encoder/decoder can concurrently handle the errors inflicted by the different noise sources depicted in Fig. 7, or multiple dedicated encoders/decoders should be considered.

\section{B. Mitigating imperfections at the Entanglement Generator \& Distributor}

We can also modify the quantum communication system model of Fig. 5 by introducing a system block for mitigating the imperfections imposed on the entanglement resources.

Indeed, provided that the contamination of the entangled qubits is below a certain threshold, it is possible to purify multiple imperfectly entangled pairs into a single "almostmaximally entangled" pair, albeit only at the price of additional processing. This strategy is known as entanglement distillation or purification [97], and it has been lavishly documented in the literature [98]-[100].

Furthermore, the entanglement distribution rate decays exponentially with the distance between Alice and Bob [75], 
[101]. As highlighted in Table [II, no classical strategies such as amplify-and-forward or decode-and-forward can be employed. Instead, we must use quantum repeaters [21], [67], [70], [74], [102], [103] that implement entanglement swapping [66], which allows us to entangle qubits over a long link by generating and distributing entanglement through shorter links. In practice, two EPR pairs are generated and distributed. One pair between Alice and an intermediate node - namely the quantum repeater - and another pair between the intermediate node and Bob. By performing a BSM on the two particles at the quantum repeater, entanglement is created between the particles at Alice and Bob [1], [5]. Clearly, the decoherencecontaminated entanglement swapping procedure itself also introduces errors [104], [105], which can be tackled either by entanglement purification or by QEC techniques [21].

However, further research is needed. In fact, the quantum encoder/decoder blocks at the Quantum Pre/Post-Processing blocks and the ones at the Entanglement Generator \& Distributor can be separately designed, although a joint - but much more difficult - design is also conceivable. However, it is unclear whether the joint design provides superior performance.

In conclusion, a substantial amount of frontier-research is required for tackling the challenges and open problems associated with the Quantum Internet.

However, the excitement in contributing to this research area is intoxicating, since the Quantum Internet might pave the way for the Internet of the future, such as Arpanet had paved the way for today's Internet. This is an exciting era for quantum communications and signal processing.

\section{REFERENCES}

[1] A. S. Cacciapuoti, M. Caleffi, F. Tafuri, F. S. Cataliotti, S. Gherardini, and G. Bianchi, "Quantum internet: Networking challenges in distributed quantum computing," IEEE Network, vol. 34, no. 1, pp. 137-143, January 2020.

[2] L. Hanzo, H. Haas, S. Imre, D. O'Brien, M. Rupp, and L. Gyongyosi, "Wireless Myths, Realities, and Futures: From 3G/4G to Optical and Quantum Wireless,' Proceedings of the IEEE, vol. 100, no. Special Centennial Issue, pp. 1853-1888, May 2012.

[3] M. Caleffi, A. S. Cacciapuoti, and G. Bianchi, "Quantum Internet: From Communication to Distributed Computing!' in NANOCOM '18, 2018 pp. $1-4$.

[4] H. V. Nguyen, Z. Babar, D. Alanis, P. Botsinis, D. Chandra, M. A. M Izhar, S. X. Ng, and L. Hanzo, "Towards the Quantum Internet: Generalised Quantum Network Coding for Large-Scale Quantum Communication Networks," IEEE Access, vol. 5, pp. 17 288-17 308, 2017.

[5] R. Van Meter, Quantum Networking. Wiley-ISTE, Apr. 2014.

[6] S. Pirandola and S. L. Braunstein, "Physics: Unite to Build a Quantum Internet," Nature, vol. 532, no. 7598, pp. 169-171, Apr. 2016.

[7] E. Gibney, "Chinese Satellite is One Giant Step for the Quantum Internet," Nature, vol. 535, no. 7613, pp. 478-479, July 2016.

[8] C. Simon, "Towards a Global Quantum Network," Nature Photonics, vol. 11, no. 11, pp. 678-680, 2017.

[9] W. Dür, R. Lamprecht, and S. Heusler, "Towards a Quantum Internet,' European Journal of Physics, vol. 38, no. 4, p. 043001, May 2017.

[10] D. Castelvecchi, "The Quantum Internet has Arrived (and it hasn't)," Nature, vol. 554, Feb. 2018.

[11] S. Wehner, D. Elkouss, and R. Hanson, "Quantum Internet: a Vision for the Road Ahead," Science, vol. 362, no. 6412, 2018.

[12] A. Broadbent, J. Fitzsimons, and E. Kashefi, "Universal Blind Quantum Computation," in 50th Annual IEEE Symposium on Foundations of Computer Science, Oct. 2009, pp. 517-526.
[13] Y.-B. Sheng and L. Zhou, "Distributed secure quantum machine learning," Science Bulletin, vol. 62, no. 14, pp. 1025 - 1029, 2017.

[14] - "Blind quantum computation with a noise channel," Phys. Rev A, vol. 98, Nov 2018 .

[15] C. L. Degen, F. Reinhard, and P. Cappellaro, "Quantum Sensing," Rev Mod. Phys., vol. 89, July 2017.

[16] IOT Analytics, "State of the IoT Q3/Q4 2018 \& Short-term Outlook 2019,” Tech. Rep., Mar. 2019.

[17] M. A. Nielsen and I. L. Chuang, Quantum Computation and Quantum Information. Cambridge University Press, 2011.

[18] E. Rieffel and W. Polak, Quantum Computing: A Gentle Introduction The MIT Press, 2011.

[19] R. Qi, Z. Sun, Z. Lin et al., "Implementation and security analysis of practical quantum secure direct communication," Light: Science \& Applications, vol. 8, 2019.

[20] C. H. Bennett, D. P. DiVincenzo, J. A. Smolin, and W. K. Wootters, "Mixed-state entanglement and quantum error correction," Phys. Rev. $A$, vol. 54, no. 5, pp. 3824-3851, Nov. 1996

[21] S. Muralidharan, L. Li, J. Kim, N. Lütkenhaus et al., "Optimal architectures for long distance quantum communication," Scientific Reports, vol. 6, p. 20463, 2016.

[22] C. H. Bennett, G. Brassard, C. Crépeau et al., "Teleporting an unknown quantum state via dual classical and Einstein-Podolsky-Rosen channels," Physical Review Letters, vol. 70, pp. 1895-1899, Mar. 1993.

[23] L. Gyongyosi, S. Imre, and H. V. Nguyen, "A Survey on Quantum Channel Capacities," IEEE Comm. Surveys Tutorials, vol. 20, no. 2, 2018.

[24] X.-M. Hu, C. Zhang, C.-J. Zhang et al., "Experimental certification for nonclassical teleportation," Ouantum Engineering, vol. 1, no. 2, 2019.

[25] J.-G. Ren, P. Xu, H.-L. Yong et al., "Ground-to-Satellite Quantum Teleportation," Nature, vol. 549, pp. 70 EP -, 082017.

[26] C. E. Shannon, "A Mathematical Theory of Communication," Bell System Technical Journal, vol. 27, no. 3, pp. 379-423, 1948.

27] H. V. Nguyen, Z. Babar, D. Alanis, P. Botsinis, D. Chandra, S. X. Ng, and L. Hanzo, "EXIT-Chart Aided Quantum Code Design Improves the Normalised Throughput of Realistic Quantum Devices," IEEE Access, vol. 4, pp. 10 194-10209, 2016.

[28] P. Botsinis, D. Alanis, Z. Babar, H. V. Nguyen, D. Chandra, S. X. Ng, and L. Hanzo, "Quantum Search Algorithms for Wireless Communications," IEEE Communications Surveys Tutorials, vol. 21, no. 2, pp. 1209-1242, 2019

[29] D. Castelvecchi, "IBM's Quantum Cloud Computer goes Commercial," Nature, vol. 543, no. 7644, p. 159, Mar. 2017.

[30] Z. Babar, D. Chandra, H. V. Nguyen, P. Botsinis, D. Alanis, S. X $\mathrm{Ng}$, and L. Hanzo, "Duality of Quantum and Classical Error Correction Codes: Design Principles and Examples," IEEE Communications Surveys Tutorials, vol. 21, no. 1, pp. 970-1010, 2019.

[31] P. Benioff, "The computer as a physical system: A microscopic quantum mechanical hamiltonian model of computers as represented by turing machines," Journal of Statistical Physics, vol. 22, no. 5, pp. 563-591, May 1980.

[32] L. Gui-Lu, "General quantum interference principle and duality computer," Communications in Theoretical Physics, vol. 45, no. 5, pp. 825844, may 2006

[33] D. Bohm and Y. Aharonov, "Discussion of experimental proofs for the paradox of einstein, rosen and podolsky," Phys. Rev., vol. 108, pp. 1070-1076, 1957.

[34] A. Einstein, B. Podolsky, and N. Rosen, "Can Quantum-Mechanical Description of Physical Reality Be Considered Complete?" Physical Review, vol. 47, pp. 777-780, May 1935

[35] R. Horodecki, P. Horodecki, M. Horodecki, and K. Horodecki, "Quantum entanglement," Rev. Mod. Phys., vol. 81, pp. 865-942, June 2009.

[36] J. F. Clauser, M. A. Horne, A. Shimony, and R. A. Holt, "Proposed experiment to test local hidden-variable theories," Phys. Rev. Lett. vol. 23 , pp. 880-884, Oct. 1969.

[37] M. A. Schlosshauer, Decoherence and the Quantum-To-Classical Transition. Springer-Verlag Berlin Heidelberg, 2007.

[38] R. A. Horn and C. R. Johnson, Matrix Analysis, 2nd ed. Cambridge University Press, 2012

[39] J. L. Park, "The Concept of Transition in Quantum Mechanics," Foundations of Physics, vol. 1, no. 1, pp. 23-33, Mar. 1970.

[40] W. K. Wootters and W. H. Zurek, "A Single Quantum cannot be Cloned," Nature, vol. 299, no. 5886, pp. 802-803, 1982.

[41] D. Dieks, "Communication by EPR devices," Physics Letters A, vol. 92 no. 6 , pp. $271-272,1982$

[42] J. von Neumann, Mathematical Foundations of Quantum Mechanics. Princeton University Press, 1955. 
[43] L. Landau, "Das dämpfungsproblem in der wellenmechanik," Zeitschrift für Physik, vol. 45, no. 5, May 1927.

[44] B. D'espagnat, Veiled Reality - An Analysis Of Present- Day Quantum Mechanical Concepts. CRC Press, 2003.

[45] G. L. Long, Y.-F. Zhou, J.-Q. Jin, Y. Sun, and H.-W. Lee, "Density matrix in quantum mechanics and distinctness of ensembles having the same compressed density matrix," Foundations of Physics, vol. 36 no. 8, pp. 1217-1243, Aug 2006

[46] R. Jozsa, "Fidelity for Mixed Quantum States," Journal of Modern Optics, vol. 41, pp. 2315-2323, 1994.

[47] C. H. Bennett, D. P. DiVincenzo, P. W. Shor et al., "Remote State Preparation," Phys. Rev. Lett., vol. 87, p. 077902, July 2001.

[48] C. H. Bennett, P. Hayden, D. W. Leung et al., "Remote Preparation of Quantum States," IEEE Trans. on Information Theory, vol. 51, no. 1 pp. 56-74, Jan. 2005

[49] D. Girolami, "How difficult is it to prepare a quantum state?" ArXiv e-prints, Jan. 2019.

[50] S. L. Braunstein, C. A. Fuchs, and H. J. Kimble, "Criteria for continuous-variable quantum teleportation," Journal of Modern Optics, vol. 47 , no. $2-3$, pp. $267-278,2000$.

[51] W. P. Bowen, N. Treps, B. C. Buchler, R. Schnabel, T. C. Ralph, H.A. Bachor, T. Symul, and P. K. Lam, "Experimental investigation of continuous-variable quantum teleportation," Phys. Rev. A, vol. 67, Mar 2003.

[52] S. Pirandola and S. Mancini, "Quantum teleportation with continuous variables: A survey," Laser Physics, vol. 16, no. 10, pp. 1418-1438, Oct. 2006.

[53] P. G. Kwiat, K. Mattle, H. Weinfurter, A. Zeilinger, A. V. Sergienko, and Y. Shih, "New High-Intensity Source of Polarization-Entangled Photon Pairs," Phys. Rev. Lett., vol. 75, pp. 4337-4341, Dec. 1995.

[54] J. I. Cirac, P. Zoller, H. J. Kimble et al., "Quantum State Transfer and Entanglement Distribution among Distant Nodes in a Quantum Network," Phys. Rev. Lett., vol. 78, pp. 3221-3224, Apr. 1997.

[55] D. N. Matsukevich, T. Chanelière, S. D. Jenkins, and other, "Entanglement of Remote Atomic Qubits," Phys. Rev. Lett., vol. 96, p. 030405 , Jan. 2006.

[56] S. Ritter, C. Nölleke, C. Hahn et al., "An Elementary Quantum Network of Single Atoms in Optical Cavities," Nature, vol. 484, pp. 195-200, Apr. 2012.

[57] S. Welte, B. Hacker et al., "Photon-Mediated Quantum Gate between Two Neutral Atoms in an Optical Cavity," Phys. Rev. X, vol. 8, Feb. 2018.

[58] C. Ferrari and B. Braunecker, "Entanglement, which-way measurements, and a quantum erasure," American Journal of Physics, vol. 78 no. 8, pp. 792-795, 2010.

[59] T. E. Northup and R. Blatt, "Quantum information transfer using photons," Nature Photonics, vol. 8, pp. 356 EP -, Apr. 2014.

[60] M. Caleffi, "Optimal Routing for Cavity-Based Quantum Networks," IEEE Access, vol. 5, pp. 22 299-22 312, 2017.

[61] S. Olmschenk, D. N. Matsukevich, P. Maunz et al., "Quantum Teleportation Between Distant Matter Qubits,” Science, vol. 323, no. 5913 , pp. 486-489, 2009.

[62] C. Jones, D. Kim, M. T. Rakher et al., "Design and analysis of communication protocols for quantum repeater networks," New Journal of Physics, vol. 18, no. 8, p. 083015, 2016.

[63] M. Afzelius, N. Gisin, and H. De Riedmatten, "Quantum memory for photons," Phys. Today, vol. 68, no. 12, pp. 42-47, 2015.

[64] F. Flamini, N. Spagnolo, and F. Sciarrino, "Photonic Quantum Information Processing: a Review," Reports on Progress in Physics, vol. 82 no. 1, p. 016001 , Nov. 2018.

[65] R.-B. Jin, R. Shimizu, K. Wakui, M. Fujiwara, T. Yamashita, S. Miki, H. Terai, Z. Wang, and M. Sasaki, "Pulsed sagnac polarizationentangled photon source with a ppktp crystal at telecom wavelength,' Opt. Express, vol. 22, no. 10, pp. 11 498-11 507, May 2014.

[66] M. Żukowski, A. Zeilinger, M. A. Horne et al., "'Event-readydetectors' Bell Experiment via Entanglement Swapping," Physical Review Letters, vol. 71, pp. 4287-4290, Dec. 1993.

[67] R. Van Meter, T. D. Ladd, W. J. Munro et al., "System Design for a Long-Line Quantum Repeater," IEEE/ACM Trans. on Networking, vol. 17 , no. 3, pp. 1002-1013, June 2009

[68] H. Bernien, B. Hensen, W. Pfaff et al., "Heralded entanglement between solid-state qubits separated by three metres," Nature, Apr. 2013.

[69] P. Kurpiers, P. Magnard, T. Walter et al., "Deterministic quantum state transfer and remote entanglement using microwave photons," Nature, vol. 558, pp. 264-267, 2018.
[70] L. M. Duan, M. D. Lukin, J. I. Cirac, and P. Zoller, "Long-distance quantum communication with atomic ensembles and linear optics," Nature, vol. 414, 2001.

[71] D. Chandra, Z. Babar, H. V. Nguyen, D. Alanis, P. Botsinis, S. X. Ng, and L. Hanzo, "Quantum Topological Error Correction Codes: The Classical-to-Quantum Isomorphism Perspective," IEEE Access, vol. 6, pp. 13 729-13 757, 2018

[72] D. P. DiVincenzo, "The Physical Implementation of Quantum Computation," Fortschritte der Physik, vol. 48, no. 9/11, pp. 771-783, 2000

[73] S. J. Devitt, A. D. Greentree, A. M. Stephens, and R. Van Meter "High-speed Quantum Networking by Ship," Scientific Reports, vol. 6 , p. 36163,2016

[74] H.-J. Briegel, W. Dür, J. I. Cirac, and P. Zoller, "Quantum Repeaters: The Role of Imperfect Local Operations in Quantum Communication," Physical Review Letters, vol. 81, pp. 5932-5935, Dec. 1998.

[75] S. Pirandola, R. Laurenza, C. Ottaviani, and L. Banchi, "Fundamental limits of repeaterless quantum communications," Nature communications, vol. 8, p. 15043, 2017.

[76] J. G. Proakis, Digital Communications. McGraw-Hill, 2001

[77] D. A. Lidar and T. A. Brun, "Introduction to decoherence and noise in open quantum systems," in Quantum Error Correction. Cambridge Press, 2013, pp. 3-45.

[78] "IBM Q Experience." [Online]. Available: https://www.research.ibm. com/ibm-q/

[79] S.-J. Wei, D. Ruan, and G.-L. Long, "Duality quantum algorithm efficiently simulates open quantum systems," Scientific Reports, vol. 6 , no. $1,2016$.

[80] S. Oh, S. Lee, and H.-w. Lee, "Fidelity of quantum teleportation through noisy channels," Phys. Rev. A, vol. 66, p. 022316, Aug. 2002.

[81] M. J. Biercuk, A. C. Doherty, and H. Uys, "Dynamical decoupling sequence construction as a filter-design problem," Journal of Physics B: Atomic, Molecular and Optical Physics, vol. 44, no. 15, p. 154002, 2011

[82] A. S. Cacciapuoti and M. Caleffi, "Toward the Quantum Internet: A Directional-dependent Noise Model for Quantum Signal Processing," in ICASSP'19, 2019.

[83] J. M. Chow, J. M. Gambetta, A. D. Córcoles et al., "Universal Quantum Gate Set Approaching Fault-Tolerant Thresholds with Superconducting Qubits," Phys. Rev. Lett., vol. 109, p. 060501, Aug. 2012.

[84] J. Altepeter, E. Jeffrey, and P. Kwiat, "Photonic State Tomography," ser. Advances In Atomic, Molecular, and Optical Physics. Academic Press, 2005, vol. 52, pp. 105-159.

[85] R. Blume-Kohout, "Optimal, reliable estimation of quantum states," New Journal of Physics, vol. 12, no. 4, p. 043034, Apr. 2010.

[86] D. H. Mahler, L. A. Rozema, A. Darabi et al., "Adaptive Quantum State Tomography Improves Accuracy Quadratically," Phys. Rev. Lett. vol. 111 , p. 183601 , Oct. 2013.

[87] T. Oka, T. Satoh, and R. Van Meter, "A Classical Network Protocol to Support Distributed Quantum State Tomography," in Proc. Quantum Communications and Information Technology, Dec. 2016.

[88] H. Wang, W. Zheng, N. Yu, K. Li et al., "Quantum state and process tomography via adaptive measurements," Science China Physics, Mechanics \& Astronomy, vol. 59, no. 10, Aug 2016.

[89] S. Huang, J. Chen, Y. Li, and B. Zeng, "Quantum state tomography for generic pure states," Science China Physics, Mechanics \& Astronomy, vol. 61, no. 11, Aug 2018.

[90] K. Rudinger, T. Proctor, D. Langharst et al., "Probing ContextDependent Errors in Quantum Processors," Phys. Rev. X, vol. 9, p 021045, Jun. 2019.

[91] P. W. Shor, "Scheme for reducing decoherence in quantum computer memory," Phys. Rev. A, vol. 52, pp. R2493-R2496, Oct. 1995.

[92] D. A. Lidar, I. L. Chuang, and K. B. Whaley, "Decoherence-free subspaces for quantum computation," Phys. Rev. Lett., vol. 81, Sep 1998.

[93] D. Suter and G. A. Álvarez, "Colloquium: Protecting quantum information against environmental noise," Rev. Mod. Phys., vol. 88, Oct 2016.

[94] C. P. Koch, "Controlling open quantum systems: tools, achievements, and limitations," Journal of Physics: Condensed Matter, vol. 28, no. 21, may 2016.

[95] L. Viola, E. Knill, and S. Lloyd, "Dynamical decoupling of open quantum systems," Phys. Rev. Lett., vol. 82, Mar 1999.

[96] L. Viola and S. Lloyd, "Dynamical suppression of decoherence in twostate quantum systems," Phys. Rev. A, vol. 58, Oct 1998.

[97] C. H. Bennett, G. Brassard, S. Popescu et al., "Purification of Noisy Entanglement and Faithful Teleportation via Noisy Channels," Phys. Rev. Lett., vol. 76, pp. 722-725, Jan. 1996. 
[98] W. Dür and H. J. Briegel, "Entanglement Purification and Quantum Error Correction," Reports on Progress in Physics, vol. 70, no. 8, pp. 1381-1424, July 2007.

[99] Y.-B. Sheng and F.-G. Deng, "One-step deterministic polarizationentanglement purification using spatial entanglement," Phys. Rev. A, vol. 82, Oct 2010.

[100] - "Deterministic entanglement purification and complete nonlocal bell-state analysis with hyperentanglement," Phys. Rev. A, vol. 81, Mar 2010.

[101] M. Takeoka, S. Guha, and M. M. Wilde, "Fundamental rate-loss tradeoff for optical quantum key distribution," Nature communications vol. 5, 2014.

[102] W. Dür, H.-J. Briegel, J. I. Cirac, and P. Zoller, "Quantum Repeaters Based on Entanglement Purification," Physical Review A, vol. 59, pp. 169-181, Jan. 1999.

[103] R. V. Meter and J. Touch, "Designing Quantum Repeater Networks," IEEE Communications Magazine, vol. 51, no. 8, pp. 64-71, Aug. 2013.

[104] W. J. Munro, R. Van Meter, S. G. R. Louis, and K. Nemoto, "HighBandwidth Hybrid Quantum Repeater," Phys. Rev. Lett., vol. 101, p. 040502, July 2008

[105] L. Jiang, J. M. Taylor, K. Nemoto, W. J. Munro, R. Van Meter, and M. D. Lukin, “Quantum Repeater with Encoding,” Phys. Rev. A, vol. 79, p. 032325, Mar. 2009.

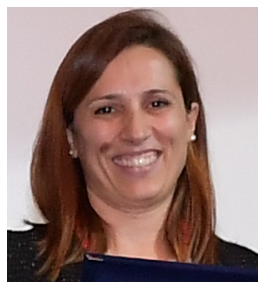

Angela Sara Cacciapuoti (M'10, SM'16) is Tenure-Track Assistant Professor at the University of Naples Federico II, Italy. Since July 2018, she held the national habilitation as "Full Professor" in Telecommunications Engineering. Currently, Angela Sara serves as Area Editor for IEEE Communications Letters, and as Editor/Associate Editor for the journals: IEEE Trans. on Communications, IEEE Trans. on Wireless Communications, IEEE Open Journal of Communications Society and IEEE Trans. on Quantum Engineering. She was a recipient of the 2017 Exemplary Editor Award of the IEEE Communications Letters. In 2016 she has been an appointed member of the IEEE ComSoc Young Professionals Standing Committee. From 2017 to 2018, she has been the Award Co-Chair of the N2Women Board. Since 2017, she has been an elected Treasurer of the IEEE Women in Engineering (WIE) Affinity Group of the IEEE Italy Section. Since 2018, she has been appointed as Publicity Chair of the IEEE ComSoc Women in Communications Engineering (WICE) Standing Committee. Her current research interests are mainly in Quantum Communications, Quantum Networks and Quantum Information Processing.

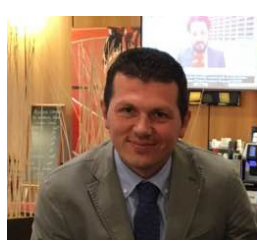

Marcello Caleffi (M'12, SM'16) is with the DIETI Department, University of Naples Federico II, and with the National Laboratory of Multimedia Communications, National Inter-University Consortium for Telecommunications (CNIT). Previously, he was with the Broadband Wireless Networking Laboratory at Georgia Institute of Technology, Atlanta, and with the NaNoNetworking Center in Catalunya (N3Cat) at the Universitat Politecnica de Catalunya (UPC), Barcelona, as visiting researcher. Since July 2018, he held the Italian national habilitation as Full Professor in Telecommunications Engineering. Currently, he serves as associate technical editor for IEEE Communications Magazine and as edito for IEEE Trans. on Quantum Engineering and IEEE Communications Letters. In 2017 he has been appointed as Distinguished Lecturer from the IEEE Computer Society and, in December 2018, he has been appointed member of the IEEE New Initiatives Committee.

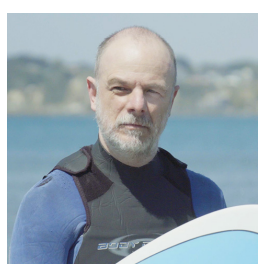

Rodney Van Meter received a B.S. in engineering and applied science from the California Institute of Technology in 1986, an M.S. in computer engineering from the University of Southern California in 1991, and a Ph.D. in computer science from Keio University in 2006. His current research centers on quantum computer architecture and quantum networking. Other research interests include storage systems, networking, and post-Moore's Law computer architecture. He is now a Professor of Environment and Information Studies at Keio University's

Shonan Fujisawa Campus. He is the Vice Center Chair of Keio's new Quantum Computing Center and the co-chair of the Internet Research Task Force's Quantum Internet Research Group. Dr. Van Meter is a member of AAAS, ACM, APS and IEEE.

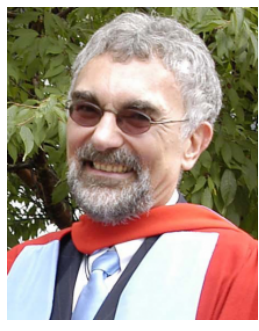

Lajos Hanzo (http://www-mobile.ecs.soton.ac.uk https://en.wikipedia.org/wiki/Lajos_Hanzo) FREng, FIEEE, FIET, Fellow of EURASIP, DSc holds an honorary doctorate by the Technical University of Budapest (2009) and by the University of Edinburgh (2015). He is a Foreign Member of the Hungarian Academy of Sciences and a former Editor-in-Chief of the IEEE Press. He has served as Governor of both IEEE ComSoc and of VTS. He has published 1900+ contributions at IEEE Xplore, 18 Wiley-IEEE Press books and has helped the fast-track career of $119 \mathrm{PhD}$ students. Over 40 of them are Professors at various stages of their careers in academia and many of them are leading scientists in the wireless industry. 